\title{
Modeling Reader's Emotional State Response on Document's Typographic Elements
}

\author{
Dimitrios Tsonos and Georgios Kouroupetroglou \\ Department of Informatics and Telecommunications, National and Kapodistrian University of Athens, \\ Panepistimiopolis, 15784 Athens, Greece \\ Correspondence should be addressed to Georgios Kouroupetroglou, koupe@di.uoa.gr
}

Received 30 July 2010; Revised 31 January 2011; Accepted 25 March 2011

Academic Editor: Kerstin S. Eklundh

Copyright ( $) 2011$ D. Tsonos and G. Kouroupetroglou. This is an open access article distributed under the Creative Commons Attribution License, which permits unrestricted use, distribution, and reproduction in any medium, provided the original work is properly cited.

\begin{abstract}
We present the results of an experimental study towards modeling the reader's emotional state variations induced by the typographic elements in electronic documents. Based on the dimensional theory of emotions we investigate how typographic elements, like font style (bold, italics, bold-italics) and font (type, size, color and background color), affect the reader's emotional states, namely, Pleasure, Arousal, and Dominance (PAD). An experimental procedure was implemented conforming to International Affective Picture System guidelines and incorporating the Self-Assessment Manikin test. Thirty students participated in the experiment. The stimulus was a short paragraph of text for which any content, emotion, and/or domain dependent information was excluded. The Analysis of Variance revealed the dependency of (a) all the three emotional dimensions on font size and font/background color combinations and (b) the Pleasure dimension on font type and font style. We introduce a set of mapping rules showing how PAD vary on the discrete values of font style and font type elements. Moreover, we introduce a set of equations describing the PAD dimensions' dependency on font size. This novel model can contribute to the automated reader's emotional state extraction in order, for example, to enhance the acoustic rendition of the documents, utilizing text-to-speech synthesis.
\end{abstract}

\section{Introduction}

Most of the current Text-to-Speech systems do not take into account the semantics and the cognitive aspects of the visual (such as font and font style) and nonvisual document elements. There is an effort towards Documentto-Audio (DtA) synthesis, which essentially constitutes the next generation of the Text-to-Speech systems, supporting the extraction of the semantics of document metadata [1] and the efficient acoustic representation of both text formatting [2-5] and data tables [6-8] through the modeling of the parameters of the synthesized speech signal by: (a) combining alternative text insertion in the document text stream, (b) altering the prosody, (c) switching between voices, and/or (d) inserting nonspeech audio (like earcons) in the waveform stream, according to the class of metadata provided in the document.
Kouroupetroglou and Tsonos [9] proposed an automated reader's emotional state extraction process derived by the typographic elements and their acoustic rendition using expressive speech synthesis. In order to facilitate the automated reader's emotional state extraction, the use of a quantitative model that describes the way typographic elements affect the reader's emotional state is mandatory.

Most of the studies (presented in the following sections) focus on the qualitative description of the way typographic elements affect the reader's emotional state and propose or suggest how the typographic elements should be used while creating a document (e.g., web pages, leaflets, and scientific documents). They do not fulfill the requirements for a more formal description using mathematical modeling in order to be used in DtA synthesis and in other systems, such as those presented in $[10,11]$. 
This work deals with the study and modeling of reader's emotional state response on typographic elements (font type/color/size and font style) during the reading process of electronic text documents. The results are expected to be utilized for the automated reader's emotional state extraction and annotation of document's typographic elements as proposed by Tsonos et al. [12]. Thus, they can be incorporated in methods and systems, such as the automated enhancement of the acoustic presentation of documents using expressive speech synthesis for the blind [13] or for people with other disabilities [14], but also in Text-to-Speech (TtS) systems for the general population (including the elderly) following a design-for-all approach $[9,10]$.

The structure of the paper is as follows: in Section 2, a background on typography and emotions is presented. Section 3 describes how the current study extends previous published work. A novel research model is introduced in Section 4 using four hypotheses, followed by the experimental procedure (Section 5). Section 6 presents the results and Section 7 how the reader's emotional state response is modeled in each hypothesis along with a discussion of the results. In the last section, some examples on how the outcomes of the present study can be applied in the HumanComputer Interaction field are pointed out.

This work extends the previous studies in the following ways. A mathematical description (model) is introduced towards automated emotional state extraction and annotation of documents' typographic elements. The results are presented in a quantitative form (and not only in qualitative, descriptive, or suggestive way). It proposes a formal way (based on the International Affective Picture System (IAPS) guidelines/Self-Assessment Manikin Test) to examine, investigate, and model the reader's emotional state response on visual presentation of documents. Moreover, the consistency of our methodology is studied compared to previous work, that is, we examine whether language and cultural aspects affect reader's emotional state response on typographic elements. The investigated typographic elements are extended (adding bold and bold-italics), and a wide range of font sizes are studied (contrary to other studies, e.g., using 2 or 3 different font sizes).

\section{Background on Typography and Emotions}

According to the communication theorist McLuhan, a document is the "medium" in which a "message" (information) is communicated [15]. Printed or electronic documents include books, newspapers, periodicals, journals, reports, articles, letters, pamphlets, webpages, and e-mails. Besides its content, a printed or electronic text document contains a number of presentation elements that apply design glyphs or typographic elements (i.e., visual representation of letters and characters in a specific font and style) and arrange the content on the page or even in the document itself. For example, the title of a chapter can be recognized by placing it at the top of the page and in larger font size than the body of the text. Also, text color can be used to indicate emphasis in a specific part of a text document.

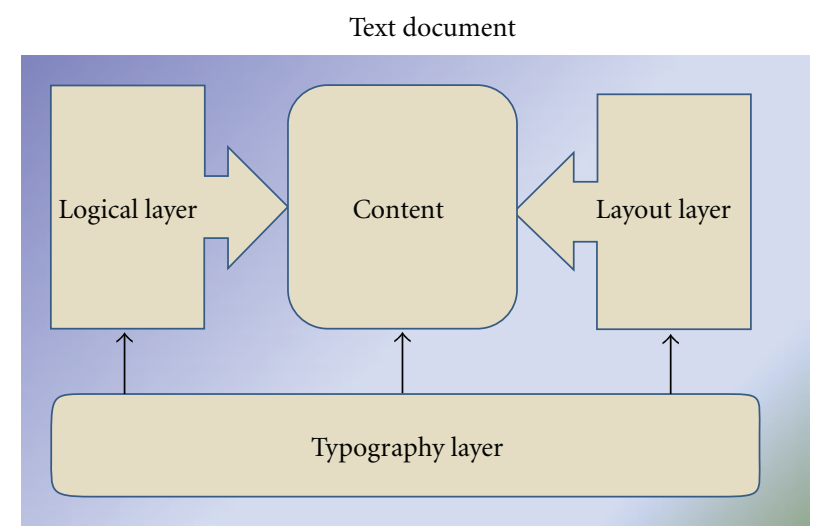

Figure 1: A general model of the text document architecture.

The presentation elements can be classified in three layers according to the general model of the text document architecture, presented in Figure 1.

(i) Logical layer: it associates content with architectural elements such as headings, titles/subtitles, chapters, paragraphs, tables, lists, footnotes, and appendices.

(ii) Layout layer: it associates content with architectural elements relating to the arrangement on pages and areas within pages, such as margins, columns, and alignment.

(iii) Typography layer: it includes font (type, size, color, background color, etc.) and font style such as bold, italics, underline. (The term "plain" used in this work indicates text of any font, but without Font Style.)

These three layers are complementary and not independent. For the scope of this work, typography (from the Greek words $\tau \hat{v} \pi \mathrm{o} \varsigma$ (typos) $=$ form and $\gamma \rho \alpha \varphi \dot{\eta}$ (graphy) $=$ writing) is the art and technique of writing words using different forms of letters. Essentially it includes font (type, size, color, background color, etc.) and font style (such as bold, italics, underline) [16]. Typography can be applied to both the logical and the layout layers of a document. For example, a footnote (Logical layer) could be in italics or in smaller font size than the body of the text. The vertical space in a text block, called leading (Layout layer), can be affected by the font type. Moreover, typography can be applied to the body of the text directly, for example, a word in bold can be used either for the introduction of a new term or to indicate a person's name.

The primary goal of utilizing presentation elements and specifically typographic elements in text documents is to distinguish parts of the text and to create a well-formed presentation of the content in order, for instance, to augment the reading performance or attract the reader. Authors use typography in a specific way. For example, there are "strict" typographic rules for the documents to be published in a scientific journal. But in newspapers and magazines the page manager, and not the authors, has the primary responsibility for applying typography. 
During the past four decades, the evolution of computer science and information technology brought out new perspectives for typography. Nowadays, the plethora of text documents are edited and stored in electronic format. Electronic documents incorporate not only presentation layers but also navigation tasks (e.g., searching for specific information in a web page). By combining the functionalities of documents and the capabilities provided by computer/information technology, the traditional concept of typography has been extended and has augmented the creation and presentation possibilities for a document. New techniques have been introduced for the document presentation in order, for example, to convey ideas, messages, and emotions or to enhance content.

Traditional factors that play a leading role in documents formation and presentation include readability (the gauge that measures how easily words, phrases, and blocks of copy can be read) and legibility (the measure of easiness to distinguish one letter from another in a particular typeface) [17]. More recent factors include visual aesthetics and accessibility.

Previous studies on emotions can be distinguished into categorical (discrete emotions) and dimensional. Ekman [18] developed a discrete emotions framework by defining six basic emotions: anger, disgust, fear, joy, sadness, and surprise. According to Bradley [19] and Bradley and Lang [20] the emotional space includes a set of three dimensions: pleasure (or valence), arousal, and dominance. Pleasure and arousal are perceived as the two most prominent dimensions [21]. The dimension of pleasure spans from negative to positive emotional pole, and its middle represents the neutral affect. The dimension of arousal varies from calm to highly aroused pole [22]. But, the interdisciplinary field of emotion studies has not yet come to an agreement on a theory of emotion.

The experimental procedures that have been developed by psychologists to assess people's/participants' emotional states include free-response measurements, forced choice response measurements, as well as other psychophysical or psychophysiological methods.

In free-response measurements the participants are asked to respond with freely chosen labels or short expressions that characterize the nature of the emotional state they experienced during a specific experimental procedure. Instructors and researchers face problems when applying this approach, like in the cases where the participants use inappropriate labels or wrong terms to express their emotions that may constrain their responses. Additionally, it is quite difficult to statistically analyze free responses [23]. Researchers tend to sort numerous free responses into a small number of emotion categories using synonyms and resemblances. This is not a standard experimental procedure, but efforts have been made towards standardizing the emotional labels.

Forced choice response measurements can be subdivided into those based on either Discrete Emotions or on Emotional Dimensions.

In the Discrete Emotions approach the participants are asked to assess their emotions by verbal expressions that best describe them on a 3- to 5-point scale or use an analogue
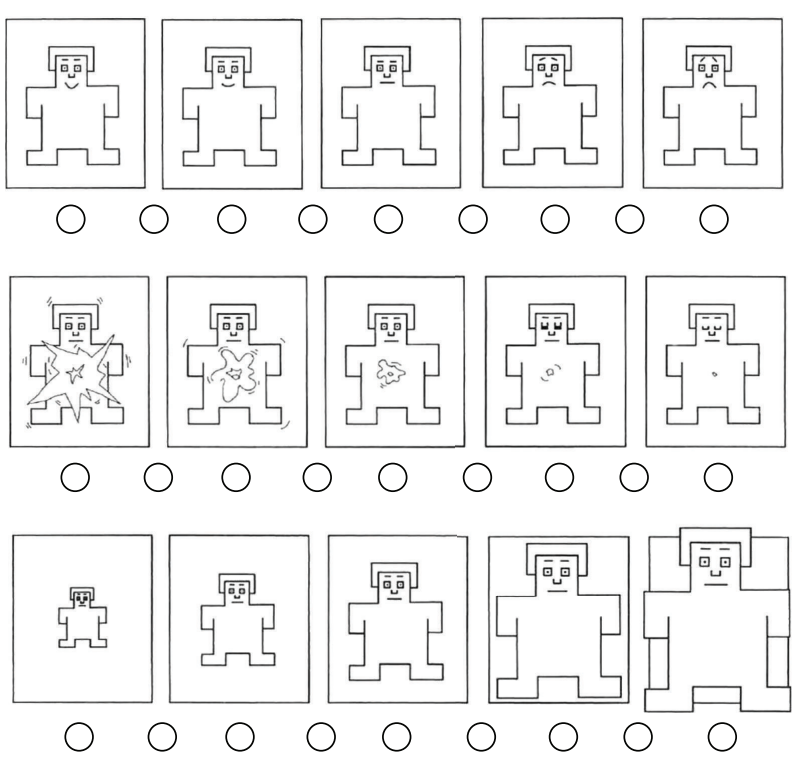

FIGURE 2: Manikins in the 9-point scale SAM test for the "Pleasure" (first row), "Arousal" (second row), and "Dominance" (third row) axes.

scale to indicate their intensity. Although there is a standardized measurement procedure for this type of experiments, many researchers prefer to develop their own emotional categories. Such methods result in mismatched categories that may present problems concerning the comparability of the results [23]. There are also difficulties in cross-study statistical analyses due to the abundance of missing data.

The participants in the Emotional Dimensions procedure are asked to denote how positive (pleasant) or negative (unpleasant) and how aroused (excited) or calm they feel. The emotions can be mapped using the bidimensional space of Pleasure-Arousal. This method seems more straightforward [23]. The bidimensional space could be estimated under a variety of conditions, to characterize features of emotion expression and their dependencies on external (e.g., environmental) and cognitive (e.g., personal significance) factors [24]. Over and above, simple or advanced singlestudy or cross-study statistical analyses can be obtained contrary to the Free Response and Discrete Emotion measurements.

Following the Dimensional approach, Lang [25] introduced the Self-Assessment Manikin test (SAM) to assess the emotional states "Pleasure," "Arousal," and "Dominance" of the participants (known also as the "PAD test"). Synonyms are used for the expression of the PAD dimensions. "Pleasure" can be replaced by "valence" or "evaluation," "Arousal" by "activation" or "activity," "Dominance" by "power" or "potency." The participants can choose between at least 5 selections of manikins. There are no verbal expressions to assess their emotional states. In some studies $[12,26]$ larger scales, from 9 to 25 points, are used. For example, on the 9-point scale (Figure 2) there are 5 image selections and 4 points as interval values between them. For the emotional state of "Pleasure" the rating spans from a happy (smiling) 
manikin to an unhappy (frowning) one. For the "Arousal" dimension the one pole is represented by a highly energetic manikin and the other by a relaxed with eyes-closed one, while for "Dominance" the controlled and in-control poles are represented by a small and large manikin, respectively. The answers of the participants can be transformed from a point scale into a dimensional space of $[-1,1]$ or $[-100 \%, 100 \%]$. By using the percentage scale one can easily distinguish how much an emotional state has been varied from the neutral state (the value "zero" represents the neutral condition in all dimensions). Having a pictorial assessment, rather than a verbal one, the SAM test becomes crosscultural and language independent [26]. The SAM procedure has been broadly applied as a test for the assessment of advertisements [27].

The three-dimensional space of emotional states can describe and map the verbal expressions of discrete emotions. A well-known example is Russell's circumplex [23, 28]. The two dimensions of "Pleasure" and "Arousal" are represented on an $X-Y$ grid, respectively. Russell placed the verbal expressions of the emotions on the grid in order to present the description of the discrete emotions using the dimensional approach. Another version of emotional grid is the Geneva Emotion Wheel experimental tool $[23,29]$.

The emotional aspects of text documents are conveyed by the semantics of either their content or their typography.

Content and Emotions. Several studies focus on the semantics-based extraction and modeling of emotions from the content of documents. Boucouvalas [30] demonstrated a real-time application which enhances interpersonal communication by detecting specific emotions (happiness, sadness, anger, fear, disgust, and surprise) during text typing and displaying them on the screen of the communication partner through appropriate facial expression images. An emotion extraction engine (Text-to-Emotion engine) based on word tagging and sentence analysis has been developed by Zhe et al. [31]. Liu et al. [32] studied the affect detection in text. Wu et al. [33] presented a domain-dependent approach towards the automatic three-state (happy, unhappy, and neutral) emotional recognition from text (for the Chinese language). Min and Park [34] proposed a number of distinct features of emotions derived from text (of the Korean language) and their appropriate representation using labels, duration, and intensity. Based on virtual or physical avatars, Yang et al. [35] introduced the concept of embodying textual messages (using simplified Chinese) in order to efficiently express nonverbal information, such as human emotions embedded in the text. Gill et al. [36] examined the ability of naive raters of emotion to detect one of eight emotional categories from 50 and 200 word samples of weblog text. Hancock et al. [37] researched how people express and detect emotions during text-based communication, that is, in a manner that eliminated the nonverbal cues typically associated with emotions (such as gestures, facial expressions, body posture, and biophysical cues). Using supervised machine learning for text-based emotion prediction, Alm [38] tried to classify the emotional affinity of sentences in the narrative domain of children's fairy tales, with a view to incorporate the results in expressive $\mathrm{TtS}$ synthesis. In their study they use the specific positively and negatively surprised emotions: angry, disgusted, fearful, happy, and sad.

Read [39] proposes a novel approach, based on the language used in conjunction with emoticons, that can train a classifier and function independent of the domain, topic, and time. Cowie et al. [40] described an empirical approach to identifying the kind of task that an emotion recognition system could usefully address. Three levels of information are elicited: a basic emotion vocabulary, a basic representation in "evaluation-activation space" of the meaning of each word, and a richer "schema" representation. They concluded that the domain of emotion understanding can be represented as a network involving nodes of many kinds. Owsley et al. [41] proposed a set of techniques that can be used to classify weblogs by emotional content. Instead of using a generalpurpose emotional classification strategy, their approach aimed at generating domain-specific sentiment classifiers. Lin et al. [42] classified news articles (of Chinese language) into eight emotions invoking in their readers: happy, angry, sad, surprised, heart warming, awesome, bored, and useful.

Typography and Emotions. Legibility and readability were introduced as basic metrics of typographic clarity for both printed and electronic documents presented on a computer screen [43]. They are closely related to the typographic elements typeface and font/background color combinations $[44,45]$. Readability is more related to the overall structure or the page layout of a document [46] in English and [47-49] in Danish, Swedish, Norwegian, and Finish. Hill and Scharf [50] found that there is no one foreground/background combination, font, or font style which leads to the best readability. The aesthetics of a document play a significant role during the reading process $[51,52]$.

The effect of document structure on reading comprehension and browsing has been studied by Calisir et al. [53] for the Turkish. Text and background color combinations in a web page have impact on its readability and aesthetics $[45,50,52]$. Thus, an appropriately designed web document can be reader friendly [54]. Hall and Hanna [45] investigated the impact of text-background color combinations of web pages on readability, retention, aesthetics, and behavioral intention. A major finding is that the preferred chromatic colors led to higher ratings of the aesthetic quality. Éthier et al. [55] examine the impact of interface features from four websites on the cognitive process that triggered online shoppers' emotions, operationalized as mental states of readiness that arise from appraisal of events and considered as direct antecedents to approach or avoidance behaviors. That study focused on six specific emotions experienced during interactions with a website that takes the form of information transfer and communication processes.

Laarni [56] investigated the effects of color, font type, and font style on user preferences. He concluded that (I) the most readable combinations are (I.1) plain Times New Roman black color font on white background, (I.2) italized 
Arial white font on blue background, and (I.3) plain Arial white font on black background and (II) the least readable combinations are the red font on green background. Also, he examined the impact of color on document aesthetics (e.g., combinations of red font on green background were rated as the most unpleasant, and black on white was considered the least arousing).

Moreover, other studies showed how multiple combinations of colors [57], font size, type, and style in a document affect the reader's emotional state [58-60] and the readability of the document, not only in printed but also in electronic format [61]. TextTone [62] is a system that adds emotions to online textual communication by changing the attributes of the typographic elements. Supported emotional tones include: happy, very happy, upset, disappointed, angry, very angry, shocked, confused, winky, tongue-in-cheek, embarrassed and "none" (the default no-tone option). TextTone typographic elements embody: font size, font color, font face, and whether the font style bold, italics, underline, and strikethrough. Ohene-Djan et al. $[63,64]$ presented how the alternations of typographic elements can be used to convey emotions in subtitles, focusing on deaf and hearingimpaired people. In their study they use the font-color and font-size typographic elements and the emotions: happily, sadly, sarcastically, excitedly, comically, fearfully, pleadingly, questioningly, authoritatively, and angrily.

Yannicopoulou [65] examined how Greek preschoolers recognize multimodal conventions of analogy, like the increase of voice volume visualized by the letter size, or conventional visual metaphors refer to different emotions. Also, Rosenberger [66] introduced the "prosodic font" that takes its temporal form from the emotionally specific prosodic parameters of the speech. Actually, the prosodic font emulates the tonal and rhythmic motion of the speaking voice [67], and it is related to the dynamic and kinetic typography.

\section{How Present Work Extends Previous Research}

Laarni [56] followed the PAD approach in his study using pseudotext, but his work exhibited two major limitations: the results were restricted to the values of the emotional states for each stimulus, and he provides suggestions only on how specific typographic elements should or should not be used (such as, red font on green background color combinations should be avoided). Moreover, he studied the impact of only the plain and italics font style element, although bold and bold-italics are widely used (in some cases more frequently than italics). Also, in his study the investigation of font size is absent. Other researches (cf. [68, 69]) are investigating specific values of font size and give suggestions for their use.

In the literature there is a debate concerning the use of font type. Some believe that the use of Arial is more preferable than the use of Times New Roman [69, 70]. But others [56] consider that Times New Roman is more preferable. In the present work, we deal with how font type affects reader's emotional states, and we are adding our contribution to the above debate.

Moreover, in this study, we deploy a novel model of the readers emotion variations induced by the typographic elements font style (bold, italics, bold-italics) and font elements (font/background color combinations, size, and type).

\section{Research Hypotheses}

The research study investigated include four hypotheses $\left(H_{i}, i=1,2,3,4\right)$ for which the independent variables are

(i) font/background color combinations,

(ii) font size,

(iii) font type,

(iv) font style,

and the three emotional dimensions, "Pleasure," "Arousal," and "Dominance" constitute the dependent variables.

Our first hypothesis is as the following.

Hypothesis 1. Font/background, font size, font type, and font style affect Pleasure, Arousal, and Dominance.

We have chosen the following seven font-color on background-color combinations: yellow on blue (Y/U), white on black $(\mathrm{W} / \mathrm{B})$, white on blue $(\mathrm{W} / \mathrm{U})$, red on green $(\mathrm{R} / \mathrm{G})$, black on gray $(\mathrm{B} / \mathrm{G})$, black on white $(\mathrm{B} / \mathrm{W})$, and green on yellow $(\mathrm{G} / \mathrm{Y})$. We have selected the color combinations proposed by Hill and Scharff [50] that have been also used by Laarni [56].

In order to study how the font/background color combinations affect the emotional states using various font types and font style elements, we investigate the following combinations using $16 \mathrm{px}(5.28 \mathrm{~mm})$ font size text: plain, bold and bold-italics for the case of Times New Roman and bold, bold-italics for Arial.

Table 1 presents the font/background color combinations used in this study: (a) in the Munsell perceptual color space and (b) in RGB along with the corresponding brightness/contrast values.

The values for the color brightness and contrast were derived from the following recommendation of the World Wide Web Consortium [71, 72]:

$$
\begin{aligned}
& \text { Brightness }=\frac{\mathrm{R} \times 299+\mathrm{G} \times 587+\mathrm{B} \times 114}{1000}, \\
& \text { Contrast }=\left|\mathrm{R}_{2}-\mathrm{R}_{1}\right|+\left|\mathrm{G}_{2}-\mathrm{G}_{1}\right|+\left|\mathrm{B}_{2}-\mathrm{B}_{1}\right|,
\end{aligned}
$$

where R-red, G-green, B-blue are given in the RGB scale.

The range for the color brightness values spans from 0 to 255 and for the contrast from 0 to 765 . The W3C recommendation for the minimum value of color brightness is 125 and for color contrast is 500 . Thus, black on white, white on black, yellow on blue, and white on blue are considered as high-contrast color combinations and red on green, black on gray, and green on yellow are considered as low contrast. 
TABLE 1: The font/background color combinations used in this study: (a) in the Munsell perceptual color space and (b) in RGB along with the corresponding brightness/contrast values.

(a)

\begin{tabular}{lcc}
\hline Font/Background Color Combinations & Font color & Background color \\
\hline Black on White & $\mathrm{N} 0,0 / 0,0$ & $8,6 \mathrm{RP} 9,8 / 0,6$ \\
White on Black & $8,6 \mathrm{RP} 9,8 / 0,6$ & $\mathrm{~N} 0,0 / 0,0$ \\
Yellow on Blue & $0,2 \mathrm{GY} 9,5 / 12,7$ & $7,0 \mathrm{~PB} 3,2 / 29,4$ \\
Red on Green & $7,9 \mathrm{R} 5,2 / 20,7$ & $9,6 \mathrm{GY} 4,5 / 11,5$ \\
Black on Gray & $\mathrm{N} 0,0 / 0,0$ & $\mathrm{~N} \mathrm{5,2/0,0}$ \\
Green on Yellow & $9,6 \mathrm{GY} 4,5 / 11,5$ & $0,2 \mathrm{GY} 9,5 / 12,7$ \\
White on Blue & $8,6 \mathrm{RP} 9,8 / 0,6$ & $7,0 \mathrm{~PB} 3,2 / 29,4$ \\
\hline
\end{tabular}

(b)

\begin{tabular}{lcccccccc}
\hline \multirow{2}{*}{ Font/Background color combinations } & \multicolumn{3}{c}{ Font color } & \multicolumn{3}{c}{ Background color } & \multicolumn{2}{c}{ Color } \\
& $\mathrm{R}$ & $\mathrm{G}$ & $\mathrm{B}$ & $\mathrm{R}$ & $\mathrm{G}$ & $\mathrm{B}$ & Brightness & Contrast \\
\hline Black on White & 0 & 0 & 0 & 255 & 255 & 255 & 255 & 765 \\
White on Black & 255 & 255 & 255 & 0 & 0 & 0 & 255 & 765 \\
Yellow on Blue & 255 & 255 & 0 & 0 & 0 & 255 & 197 & 765 \\
Red on Green & 255 & 0 & 0 & 0 & 128 & 0 & 1 & 383 \\
Black on Gray & 0 & 0 & 0 & 128 & 128 & 128 & 128 & 384 \\
Green on Yellow & 0 & 128 & 0 & 255 & 255 & 0 & 151 & 382 \\
White on Blue & 255 & 255 & 255 & 0 & 0 & 255 & 226 & 510 \\
\hline
\end{tabular}

From the indicative results derived by other studies (extensively presented and discussed in Section 7), in general, high-color-contrast combinations are expected to be more pleasant, calm and decrease the dominance level.

The second hypothesis of our study is as follows.

Hypothesis 2. Font sizes of black Times New Roman text on white background affect "Pleasure," "Arousal," and "Dominance."

Bernard et al. [69] showed that text at 12 pt size on a computer display is significantly preferred compared to the $10 \mathrm{pt}$ size. Schmidt et al. [73] concluded that increasing the font size (from $7.5 \mathrm{pt}$ to $14 \mathrm{pt}$ ) causes both user preference and ease of interaction in web pages to rise. Alsumait et al. [74] investigated the Arab children's preferences while using e-learning programs. They concluded that $14 \mathrm{pt}$ font size (compared to $12 \mathrm{pt}$ ) was more preferable and the Simplified Arabic font type (easy and fast to read) and Arial (attractive) were preferred among five font types.

Preserving the same font/background color combination (black on white), font type (Times New Roman), and font style, we examine variations of the three emotional dimensions with respect to the following font sizes (in parentheses the physical height of the corresponding font size is given): $10 \mathrm{px}(3.3 \mathrm{~mm}), 11 \mathrm{px}(3.63 \mathrm{~mm}), 12 \mathrm{px}(3.96 \mathrm{~mm})$, $13 \mathrm{px}(4.29 \mathrm{~mm}), 14 \mathrm{px}(4.62 \mathrm{~mm}), 15 \mathrm{px}(4.95 \mathrm{~mm}), 18 \mathrm{px}$ $(5.94 \mathrm{~mm}), 26 \mathrm{px}(8.58 \mathrm{~mm})$, and $32 \mathrm{px}(10.56 \mathrm{~mm})$. These font sizes have been selected as the most frequently occurring, after a statistical analysis conducted on the corpus presented in [12].

Our third hypothesis is as the following.
Hypothesis 3. The font type, using plain, bold, italics or bolditalics, affects "Pleasure," "Arousal," and "Dominance."

Bernard et al. [69] studied the user preference of Arial and Times New Roman font types displayed on a computer screen. He concluded that the most preferable font type is Arial due to high level of readability on a computer screen. However, results of the same work [69] indicated that the $57 \%$ of the participants used as everyday default font 12point Times New Roman, whereas only 8.6\% used 12-point Arial. Results of Ling and van Schaik [70] revealed that participants showed a preference in Arial than in Times New Roman font type.

In contradiction, Laarni's [56] findings showed that Times New Roman with black font color on white background is considered the most readable and most pleasant. The font type Arial is considered the most pleasant and readable in the cases of "white on blue italicized" and of "white on black plain font/background color combinations." Furthermore, for a pleasant and nonarousing color/font combination he suggests the use of the following choices: (a) white text on blue background plain Times New Roman and (b) black on white italicized Times New Roman.

In our study, the investigated font type attributes are Times New Roman and Arial on either plain, bold, italics, or bold-italics text using black on white font/background color combination and by controlling for font size by not varying it among the stimuli (16 px). We have selected these specific font types as they are the two most commonly used font types from the Serif and Sans-Serif font type groups, respectively. The selection of the specific font families derived after conducting an extended statistical analysis on a corpus 
of English and Greek books and newspapers in printed and electronic formats [12]. According to this statistical analysis almost $83 \%$ of the text (black on white font/background color combination) in the body of the documents is in Times New Roman font type and only $17 \%$ is in Arial.

The rejection or not of Hypothesis 3 for each of the three dimensions will reveal (a) the dependency of the emotional states on the font type and (b) how the emotional states vary between font type alternation.

Our last hypothesis is the following.

Hypothesis 4. Font style, using Arial or Times New Roman, affects "Pleasure," "Arousal," and "Dominance."

As mentioned in Hypothesis 3, "black on white plain Times New Roman" and "black on white italicized Times New Roman" are good combination choices to induce the pleasant emotion.

In our study, we use four font style attributes: plain, bold, italics, and bold-italics with Times New Roman and Arial font types, using black on white font/background color combination and by retaining the same font size among the stimuli (16 px). We examine (a) how font style affects the three emotional states and (b) how these emotional states vary in the case they are affected.

\section{Method}

Since the variations of the emotions and the emotional states are continuous and nonextreme, we have selected the dimensional approach and the Self-Assessment Manikin test for the assessment of the emotions of the users. An automated experimental procedure was designed and implemented according to the International Affective Picture System guidelines [75] for the paper and pencil version of the SAM test. The experiment was carried out using a computer; the stimuli were presented on an LCD display, and the users' answers/assessment were recorded directly on the computer. A 17-inch Hewlett-Packard L1706 LCD monitor, with a resolution of $1024 \times 768$ and a maximum luminance of $300 \mathrm{~cd} / \mathrm{m}^{2}$, was used for the study. The luminance of the screen at the time of the tests was $140 \mathrm{~cd} / \mathrm{m}^{2}$. Room illuminance was 105 lux, measured with a digital illuminance meter (E-SUN LX-101) at eye level of the observers, in the vertical plane, and with the display turned off. The distance between the monitor center and the subjects' eyes was $70 \pm 5 \mathrm{~cm}$.

(1) The participants filled out a form with the following demographic information: age, gender, educational level, any visual loss, and the daily usage of a computer. The user also declared his/her consent to participate in the experiment.

(2) Each stimulus was represented using a specific paragraph of text with an emotionally neutral content.

(3) Each stimulus was presented to the participants in a random order for a constant duration (15 seconds).

(4) After the presentation of each stimulus the user had to follow the SAM test.
(5) The test answers were registered in such a way so that they could be easily retrieved during the analysis stage by the researchers.

The purpose of the experimental procedure was to assess the emotional states of the participants after reading a number of short text paragraphs presented on the screen in various combinations of typographic elements. The duration of each session was approximately 20 to 30 minutes.

During the experiment a researcher was present in order (a) to provide supplementary explanations on the procedure and (b) to supervise the proper execution of the experimental process.

5.1. Participants. In the experimental procedure there were 30 participants, ranging from 18 to 33 years old (mean age 25.5). They were undergraduate or postgraduate students, 16 males, and 14 females, and their native language was Greek. All of them have self-reported normal color vision and normal or corrected-to-normal visual acuity. Any prospective participants with color blindness were excluded from the experimental procedure.

5.2. Stimuli. The specific choices of the typographic elements of this study are analytically explained in Section 4. There was a compromise not to include a greater number of stimuli not to exhaust the participants. Forty-seven combinations of font and font style elements were investigated using Greek text (a paragraph with approximately 46 words) from which any content, emotion, and/or domain dependent information were excluded (the paragraph was determined as emotionally neutral in the pilot study presented in [12]). The text was analogous to one used by Hill and Scharff [50].

All the 47 stimuli were displayed in a random sequence on a 17 -inch LCD display with $1024 \times 768$ resolution, in full screen mode, using 32 bit color depth and the MS Internet Explorer Version 7. Figure 3 presents two samples of the stimuli used in the experiments.

5.3. The Manikins. The SAM test applied includes three 9-scale manikin sets for the "Pleasure," "Arousal," and "Dominance" emotional axes, respectively. Figure 2 illustrates the screen shot that was presented to each participant, after the projection of a stimulus (the words "Pleasure," "Arousal," and "Dominance" were not presented to the participants). The participants had the possibility to assess the induced emotional states (circles between manikins) for each stimulus. This approach (9-scale SAM Test) was chosen in order to achieve greater resolution on the results.

5.4. The Procedure. At the beginning of the experiment, the participants were asked to read carefully the exact instructions provided by the IAPS guidelines [75] and ask the instructor for possible clarifications. Then, they were asked to fill in the electronic form with their personal information and a declaration that they agree to take part in the experiment. Afterwards, they participated in a demo version of the experiment with a view to be familiarized with the procedure. 


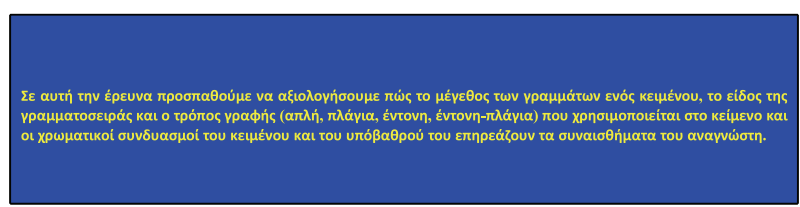

(a)

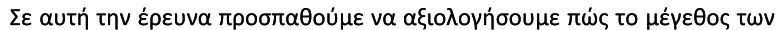

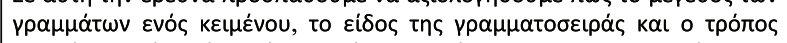

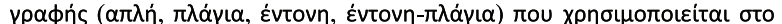

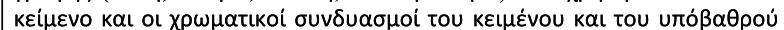

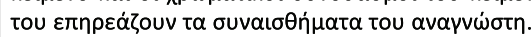

(b)

Figure 3: Sample stimuli as presented in the experimental procedure. (a) Greek text in bold Times New Roman with font size $16 \mathrm{px}$ and (b) Greek text in Times New Roman with font size 26 px. The translation of the text is: "In this study we are trying to assess how font size, font type and font style (plain, italics, bold, bold-italics) which are used in a text and the chromatic combinations of text and its background affect reader's emotions."

Each stimulus was displayed for 15 seconds, and after that, participants were asked to assess their emotional state on the 9-point PAD scale using the manikins provided by the SAM test.

Although SAM test is a rather standardized experimental procedure, there might have been some doubts on the fact the participants actually assessed their own emotional state, but we believe that their effects are included in the statistical error of the results.

\section{Results}

6.1. Hypothesis 1. The mean values and the standard error of the results of the participants for each of the 35 stimuli (numbered according to the following five groups) are presented in Figure 4.

(i) Stimuli 1-7: Plain, Times New Roman (P/TR): Y/U (Yellow on Blue), W/B (White on Black), W/U (White on Blue), R/G (Red on Green), B/G (Black on Gray), B/W (Black on White), G/Y (Green on Yellow).

(ii) Stimuli 8-14: Bold, Times New Roman (B/TR): Y/U, W/B, W/U, R/G, B/G, B/W, G/Y.

(iii) Stimuli 15-21: Bold, Arial (B/A): Y/U, W/B, W/U, R/G, B/G, B/W, G/Y.

(iv) Stimuli 22-28: Bold-Italics, Times New Roman (BI/TR): Y/U, W/B, W/U, R/G, B/G, B/W, G/Y.

(v) Stimuli 29-35: Bold-Italics, Arial (BI/A): Y/U, W/B, W/U, R/G, B/G, B/W, G/Y.

We have conducted three multifactorial repeated measures ANOVA ( 1 three-way and 2 two-way repeated measures
ANOVA) for each case of the dependent variable ("Pleasure," "Arousal," and "Dominance"). In detail:

Three-Way ANOVA. We have conducted three-way repeated measures ANOVA (for each dependent variable), using three independent variables, font/background color combinations (7 levels-chromatic combinations mentioned above), font type (2 levels-Times New Roman and Arial), and font style ( 2 levels-bold and bold-italics). The results (Table 2) are as follows.

(i) Pleasure: significant for font/background color combinations, $F(6,24)=7.668, P<.05$, font type, $F(1,29)=22.146, P<.05$, and nonsignificant for font style, $F(1,29)=0.680, P>.05$. The interaction effect is significant for font/background color combinations-font type, $F(6,24)=7.406$, $P<.05$, font/background color combinations-font style, $F(6,24)=6.436, P<.05$ and nonsignificant for font type-font style, $F(1,29)=0.785, P>$ .05. The interaction between the three variables (font/background color combinations-font typefont style) is also significant, $F(6,24)=5.213, P<.05$.

(ii) Arousal: nonsignificant for font/background color combinations, $F(6,24)=1.950, P>.05$, font type, $F(1,29)=0.321, P>.05$ and font style, $F(1,29)=$ 2.147, $P>.05$. The interaction effect is significant for font/background color combinations-font type, $F(6,24)=5.408, P<.05$, font/background color combinations-font style, $F(6,24)=4.481, P<.05$ and nonsignificant for font type-font style, $F(1,29)$ $=1.416, P>.05$. The interaction between the three variables (font/background color combinationsfont type-font style) is also significant, $F(6,24)=$ 6.051, $P<.05$.

(iii) Dominance: significant for font/background color combinations, $F(6,24)=4.945, P<.05$, font style, $F(1,29)=5.481, P<.05$, and nonsignificant for font type, $F(1,29)=2.698, P>.05$. The interaction effect is significant for font/background color combinations-font type, $F(6,24)=5.559$, $P<.05$, font/background color combinations-font style, $F(6,24)=2.929, P<.05$ and nonsignificant for font type-font style, $F(1,29)=0.334, P>$ .05. The interaction between the three variables (font/background color combinations-font typefont style) is also significant, $F(6,24)=2.917, P<.05$.

Two-Way ANOVA. We have conducted two-way repeated measures ANOVA for each dependent variable, using two independent variables, font/background color combinations (7 levels-chromatic combinations mentioned above), and font style (3 levels_-plain, bold and bold_-italics for Times New Roman and 2 levels-bold and bold-italics for Arial). The results (Table 3 ) are as follows. 


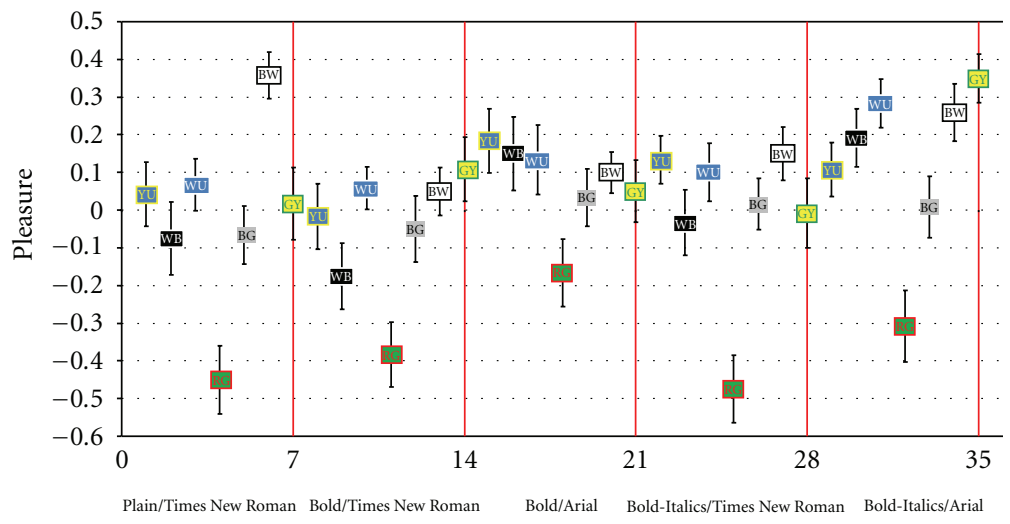

Number of stimulus

(a)

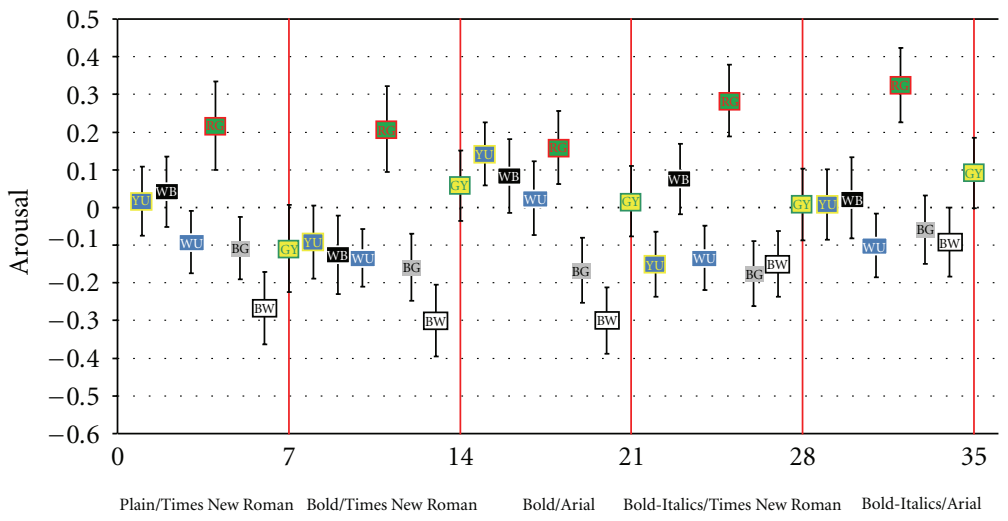

Number of stimulus

(b)

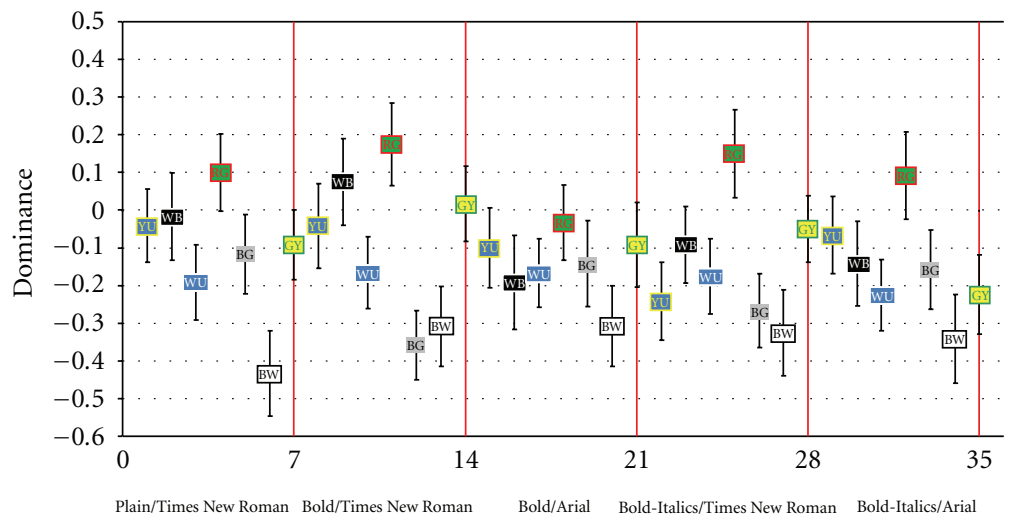

Number of stimulus

Yellow on blue

WB White on black

wu White on blue

BG Black on gray
Bw Black on white

Green on yellow

Red on green

(c)

Figure 4: (a) Mean values and standard error of the results for all the text font/background color combination stimuli on the Pleasure axis. (b) Mean values and standard error of the results for all the text font/background color combination stimuli on the Arousal axis. (c) Mean values and standard error of the results for all the text font/background color combination stimuli on the Dominance axis. 
TABLE 2: Three-way repeated measures ANOVA results for each dependent emotional dimension (PAD) on font/background color combinations, font type, font style, and their interactions.

\begin{tabular}{|c|c|c|c|}
\hline & $F$ & $P$ & $\mathrm{MSE}^{\dagger}$ \\
\hline \multicolumn{4}{|l|}{ Pleasure } \\
\hline $\begin{array}{l}\text { Font/Background } \\
\text { Color }\end{array}$ & $F(6,24)=7.668$ & $P<.05^{*}$ & 0.155 \\
\hline Font Type & $F(1,29)=22.146$ & $P<.05^{*}$ & 0.111 \\
\hline Font Style & $F(1,29)=0.680$ & $P>.05^{* *}$ & 0.126 \\
\hline $\begin{array}{l}\text { Font/Background } \\
\text { Color } * \text { Font Type }\end{array}$ & $F(6,24)=7.406$ & $P<.05^{*}$ & 0.281 \\
\hline $\begin{array}{l}\text { Font/Background } \\
\text { Color } * \text { Font Style }\end{array}$ & $F(6,24)=6.436$ & $P<.05^{*}$ & 0.191 \\
\hline $\begin{array}{l}\text { Font Type } * \text { Font } \\
\text { Style }\end{array}$ & $F(1,29)=0.785$ & $P>.05^{* *}$ & 0.085 \\
\hline $\begin{array}{l}\text { Font/Background } \\
\text { Color } * \text { Font Type } \\
* \text { Font Style }\end{array}$ & $F(6,24)=5.213$ & $P<.05^{*}$ & 0.252 \\
\hline \multicolumn{4}{|l|}{ Arousal } \\
\hline $\begin{array}{l}\text { Font/Background } \\
\text { Color }\end{array}$ & $F(6,24)=1.950$ & $P>.05^{* *}$ & 0.200 \\
\hline Font Type & $F(1,29)=0.321$ & $P>.05^{* *}$ & 0.112 \\
\hline Font Style & $F(1,29)=2.147$ & $P>.05^{* *}$ & 0.151 \\
\hline $\begin{array}{l}\text { Font/Background } \\
\text { Color } * \text { Font Type }\end{array}$ & $F(6,24)=5.408$ & $P<.05^{*}$ & 0.290 \\
\hline $\begin{array}{l}\text { Font/Background } \\
\text { Color } * \text { Font Style }\end{array}$ & $F(6,24)=4.481$ & $P<.05^{*}$ & 0.404 \\
\hline $\begin{array}{l}\text { Font Type } * \text { Font } \\
\text { Style }\end{array}$ & $F(1,29)=1.416$ & $P>.05^{*}$ & 0.076 \\
\hline $\begin{array}{l}\text { Font/Background } \\
\text { Color } * \text { Font Type } \\
* \text { Font Style }\end{array}$ & $F(6,24)=6.051$ & $P<.05^{*}$ & 0.212 \\
\hline \multicolumn{4}{|l|}{ Dominance } \\
\hline $\begin{array}{l}\text { Font/Background } \\
\text { Color }\end{array}$ & $F(6,24)=4.945$ & $P<.05^{*}$ & 0.129 \\
\hline Font Type & $F(1,29)=2.698$ & $P>.05^{* *}$ & 0.096 \\
\hline Font Style & $F(1,29)=5.481$ & $P<.05^{*}$ & 0.090 \\
\hline $\begin{array}{l}\text { Font/Background } \\
\text { Color } * \text { Font Type }\end{array}$ & $F(6,24)=5.559$ & $P<.05^{*}$ & 0.287 \\
\hline $\begin{array}{l}\text { Font/Background } \\
\text { Color } * \text { Font Style }\end{array}$ & $F(6,24)=2.929$ & $P<.05^{*}$ & 0.213 \\
\hline $\begin{array}{l}\text { Font Type } * \text { Font } \\
\text { Style }\end{array}$ & $F(1,29)=0.334$ & $P>.05^{* *}$ & 0.112 \\
\hline $\begin{array}{l}\text { Font/Background } \\
\text { Color } * \text { Font Type } \\
* \text { Font Style }\end{array}$ & $F(6,24)=2.917$ & $P<.05^{*}$ & 0.304 \\
\hline
\end{tabular}

${ }^{*}$ Statistically significant, ${ }^{* *}$ statistically nonsignificant, ${ }^{\dagger}$ Mean Square Error.

\section{Pleasure}

(i) Times New Roman: significant for font/background color combinations, $F(6,24)=5.552, P<.05$, and nonsignificant for font style, $F(2,28)=0.265$, $P>.05$. The interaction effect is significant for font/background color combinations-font style, $F(12,18)=8.749, P<.05$.
TABle 3: Two-way repeated measures ANOVA results for each dependent emotional dimension (PAD) on Times New Roman or Arial with font/background color combinations, font style, and their interactions.

\begin{tabular}{|c|c|c|c|}
\hline & $F$ & $P$ & $\mathrm{MSE}^{\dagger}$ \\
\hline \multicolumn{4}{|l|}{ Pleasure } \\
\hline \multicolumn{4}{|l|}{ Times New Roman } \\
\hline $\begin{array}{l}\text { Font/Background } \\
\text { Color }\end{array}$ & $F(6,24)=5.552$ & $P<.05^{*}$ & 0.170 \\
\hline Font Style & $F(2,28)=0.265$ & $P>.05^{* *}$ & 0.118 \\
\hline $\begin{array}{l}\text { Font/Background } \\
\text { Color } * \text { Font Style }\end{array}$ & $F(12,18)=8.749$ & $P<.05^{*}$ & 0.322 \\
\hline \multicolumn{4}{|l|}{ Arial } \\
\hline $\begin{array}{l}\text { Font/Background } \\
\text { Color }\end{array}$ & $F(6,24)=9.196$ & $P<.05^{*}$ & 0.216 \\
\hline Font Style & $F(1,29)=2.312$ & $P>.05^{*}$ & 0.079 \\
\hline $\begin{array}{l}\text { Font/Background } \\
\text { Color } * \text { Font Style }\end{array}$ & $F(6,24)=2.652$ & $P<.05^{*}$ & 0.240 \\
\hline \multicolumn{4}{|l|}{ Arousal } \\
\hline \multicolumn{4}{|l|}{ Times New Roman } \\
\hline $\begin{array}{l}\text { Font/Background } \\
\text { Color }\end{array}$ & $F(6,24)=2.795$ & $P<.05^{*}$ & 0.186 \\
\hline Font Style & $F(2,28)=1.447$ & $P>.05^{* *}$ & 0.097 \\
\hline $\begin{array}{l}\text { Font/Background } \\
\text { Color } * \text { Font Style }\end{array}$ & $F(12,18)=4.397$ & $P<.05^{*}$ & 0.348 \\
\hline \multicolumn{4}{|l|}{ Arial } \\
\hline $\begin{array}{l}\text { Font/Background } \\
\text { Color }\end{array}$ & $F(6,24)=5.107$ & $P<.05^{*}$ & 0.227 \\
\hline Font Style & $F(1,29)=3.186$ & $P>.05^{* *}$ & 0.090 \\
\hline $\begin{array}{l}\text { Font/Background } \\
\text { Color } * \text { Font Style }\end{array}$ & $F(6,24)=2.953$ & $P<.05^{*}$ & 0.283 \\
\hline \multicolumn{4}{|l|}{ Dominance } \\
\hline \multicolumn{4}{|l|}{ Times New Roman } \\
\hline $\begin{array}{l}\text { Font/Background } \\
\text { Color }\end{array}$ & $F(6,24)=3.550$ & $P<.05^{*}$ & 0.149 \\
\hline Font Style & $F(2,28)=4.043$ & $P<.05^{*}$ & 0.089 \\
\hline $\begin{array}{l}\text { Font/Background } \\
\text { Color } * \text { Font Style }\end{array}$ & $F(12,18)=6.110$ & $P<.05^{*}$ & 0.325 \\
\hline \multicolumn{4}{|l|}{ Arial } \\
\hline $\begin{array}{l}\text { Font/Background } \\
\text { Color }\end{array}$ & $\mathrm{F}(6,24)=2.323$ & $P>.05^{* *}$ & 0.252 \\
\hline Font Style & $F(1,29)=1.934$ & $P>.05^{* *}$ & 0.077 \\
\hline $\begin{array}{l}\text { Font/Background } \\
\text { Color } * \text { Font Style }\end{array}$ & $F(6,24)=1.818$ & $P>.05^{* *}$ & 0.214 \\
\hline
\end{tabular}

(ii) Arial: significant for font/background color combinations, $F(6,24)=9.196, P<.05$, and nonsignificant for font style, $F(1,29)=2.312, P>.05$. The interaction effect is significant for font/background color combinations-font style, $F(6,24)=2.652, P<$ .05 . 
TABle 4: The mean values of "Pleasure" (a), "Arousal" (b), and "Dominance" (c) on font size (using black Times New Roman text on white background) with their Standard Errors.

\begin{tabular}{lcccccc}
\hline \multirow{2}{*}{ Font Size (px) } & \multicolumn{2}{c}{ Pleasure } & \multicolumn{2}{c}{ Arousal } & \multicolumn{2}{c}{ Dominance } \\
& Mean & SE & Mean & SE & Mean & SE \\
\hline 10 & -0.47 & 0.08 & 0.08 & 0.12 & 0.06 & 0.12 \\
11 & -0.30 & 0.07 & -0.05 & 0.10 & 0.04 & 0.11 \\
12 & -0.27 & 0.07 & -0.06 & 0.10 & -0.07 & 0.11 \\
13 & -0.23 & 0.08 & -0.18 & 0.10 & -0.10 & 0.11 \\
14 & -0.01 & 0.05 & -0.36 & 0.09 & -0.21 & 0.11 \\
15 & 0.13 & 0.06 & -0.37 & 0.09 & -0.40 & 0.12 \\
16 & 0.36 & 0.06 & -0.27 & 0.10 & -0.43 & 0.11 \\
18 & 0.28 & 0.08 & -0.21 & 0.11 & -0.53 & 0.11 \\
26 & 0.43 & 0.07 & 0.02 & 0.10 & -0.31 & 0.12 \\
32 & 0.20 & 0.07 & -0.03 & 0.10 & -0.22 & 0.12 \\
\hline Mean SE & & 0.07 & & 0.10 & & 0.11 \\
\hline
\end{tabular}

TAble 5: Two-way repeated measures ANOVA results for each dependent emotional dimension (PAD) on font style, font type (using black on white font/background combination and $16 \mathrm{px}$ font size), and their interactions.

\begin{tabular}{lclc}
\hline & $F$ & $P$ & MSE $^{\dagger}$ \\
\hline Pleasure & & & \\
$\quad$ Font Style & $F(3,27)=4.033$ & $P<.05^{*}$ & 0.106 \\
$\quad$ Font Type & $F(1,29)=0.477$ & $P>.05^{* *}$ & 0.066 \\
$\quad$ Font Style * Font Type & $F(3,27)=4.150$ & $P<.05^{*}$ & 0.129 \\
\hline Arousal & & & \\
$\quad$ Font Style & $F(3,27=2.970$ & $P>.05^{* *}$ & 0.154 \\
$\quad$ Font Type & $F(1,29)=0.080$ & $P>.05^{* *}$ & 0.209 \\
$\quad$ Font Style * Font Type & $F(3,27)=0.303$ & $P>.05^{* *}$ & 0.092 \\
\hline Dominance & & & \\
$\quad$ Font Style & $F(3,27)=1.431$ & $P>.05^{* *}$ & 0.100 \\
$\quad$ Font Type & $F(1,29)=0.707$ & $P>.05^{* *}$ & 0.083 \\
Font Style * Font Type & $F(3,27)=0.281$ & $P>.05^{* *}$ & 0.120 \\
\hline
\end{tabular}

${ }^{*}$ Statistical significant, ${ }^{* *}$ statistical nonsignificant, ${ }^{\dagger}$ Mean Square Error.

Arousal

(i) Times New Roman: significant for font/background color combinations, $F(6,24)=2.795, P<.05$, and nonsignificant for font style, $F(2,28)=1.447$, $P>.05$. The interaction effect is significant for font/background color combinations-font style, $F(12,18)=4.397, P<.05$.

(ii) Arial: significant for font/background color combinations, $F(6,24)=5.107, P<.05$, and nonsignificant for font style, $F(1,29)=3.186, P>.05$. The interaction effect is significant for font/background color combinations-font style, $F(6,24)=2.953, P<$ .05 .
TABLE 6: Mean values of font style and the percentage variations from plain text using Arial (a) or Times New Roman (b) on "Pleasure" with their Standard Errors.

(a)

\begin{tabular}{|c|c|c|c|}
\hline \multicolumn{4}{|c|}{ Arial } \\
\hline & Mean & $\%$ variation from plain & SE of Mean \\
\hline Plain & 0.13 & - & 0.05 \\
\hline Bold & 0.10 & -3 & 0.05 \\
\hline Italics & 0.33 & 20 & 0.07 \\
\hline Bold-Italics & 0.26 & 13 & 0.08 \\
\hline
\end{tabular}

(b)

\begin{tabular}{lccc}
\hline & \multicolumn{2}{c}{ Times New Roman } \\
\%ean & \%ariation from plain & SE of Mean \\
\hline Plain & 0.36 & - & 0.06 \\
Bold & 0.05 & -31 & 0.06 \\
Italics & 0.17 & -19 & 0.06 \\
Bold-Italics & 0.15 & -21 & 0.07 \\
\hline
\end{tabular}

\section{Dominance}

(i) Times New Roman: significant for font/background color combinations, $F(6,24)=3.55, P<.05$, and for font style, $F(2,28)=4.043, P<.05$. The interaction effect is significant for font/background color combinations-font style, $F(12,18)=6.11, P<$ .05 .

(ii) Arial: nonsignificant for font/background color combinations, $F(6,24)=2.323, P>.05$, and for font style, $F(1,29)=1.934, P>.05$. The interaction effect is nonsignificant for font/background color combinations—font style, $F(6,24)=1.818, P>.05$.

In general, we observe that ANOVA yielded an effect for all three dimensions (namely, "Pleasure," "Arousal," and "Dominance") by the font/background color combinations.

In Section 7 we further extend the discussion of the results according to $\mathrm{H} 1 \mathrm{a}, \mathrm{H} 1 \mathrm{~b}$, and $\mathrm{H} 1 \mathrm{c}$.

6.2. Hypothesis 2. One-way repeated measures ANOVA was conducted (for each emotional state) to determine whether or not there are significant differences in the mean emotional state scores while changing the font size (10 levels).

(i) "Pleasure": The results were significant, $F(9,21)=$ $21.382, P<.05$ (Mean Square Error $=0,328$ ).

(ii) "Arousal": The results were significant, $F(9,21)=$ $3.359, P<.05$ (Mean Square Error $=0,398$ ).

(iii) "Dominance": The results were significant, $F(9,21)=$ $6.092, P<.05$ (Mean Square Error $=0,354$ ).

Thus, the font size does affect emotional dimensions and Hypothesis 2 is verified on "Pleasure" (H2a), "Arousal" (H2b), and "Dominance" (H2c). 
TABLE 7: Polynomial coefficients for the modeling of PAD relationship on text size using (2) with their standard errors.

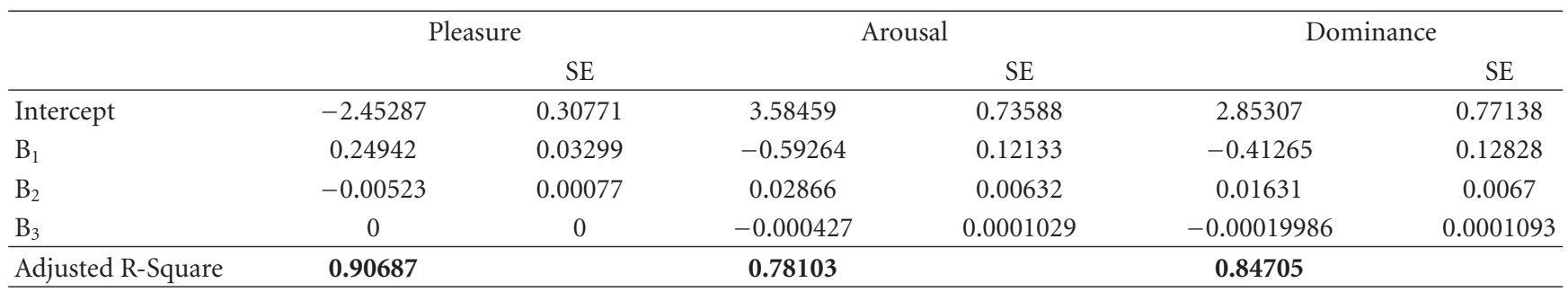

Follow-up tests using pairwise comparison (Tukey test) denoted significant differences between font sizes, for example, for font size $10 \mathrm{px}$ and $14 \mathrm{px}$, the mean values of Pleasure are significantly different $(P=.000121<.05)$.

Table 4 presents the mean values of the answers on each size along with their standard errors. In detail, we observe that while increasing the font size, the "Pleasure" dimension is increasing up to $26 \mathrm{px}$. For values greater than $26 \mathrm{px}$, there is an inclination to be diminished. In contradiction, the value of the "Arousal" dimension is decreasing up to $15 \mathrm{px}$ font size and the "Dominance" dimension up to $18 \mathrm{px}$. Beyond these values there is a tendency for the "Arousal" and "Dominance" to be increased.

6.3. Hypotheses 3 and 4. Two-way repeated measures ANOVA was conducted to determine whether or not there are significant differences in the mean emotional state scores when changing the font type (Arial and Times New Roman-2 levels) using different font style elements (plain, bold, italics, and bold-italics_-4 levels) using black font color on white background with $16 \mathrm{px}$ font size: the results are presented in Table 5.

The measured variations for Hypothesis 3 (plain text) are as follows:

(i) Arial-Pleasure: Mean $=0.13(\mathrm{SE}$ of Mean $=0.05)$.

(ii) Times New Roman-Pleasure: Mean $=0.36$ (SE of Mean $=0.06)$.

The interaction revealed that using Times New Roman instead of Arial on plain text increases pleasantness by $23 \%$. All other cases ("Pleasure" using bold, italics, bold-italics, "Arousal" using plain, bold, italics, bold-italics, and "Dominance" using plain, bold, italics, bold-italics) are statistically nonsignificant. Thus, Hypothesis $\mathrm{H} 3$ is supported only for the case of plain text on "Pleasure" (H3a).

Hypothesis 4 (H4) was not verified for the cases of "Arousal" (H4b) and "Dominance" (H4c). The ANOVA revealed the affection of font style elements on "Pleasure" (H4a).

The above results forced us to distinguish the variations of font style, while using Arial and Times New Roman, as follows (Tables 6a and 6b, resp.).

Arial. Considering the plain text as the baseline we can observe that the use of bold text marginally decreases the "Pleasure" dimension by 3\%, while italics and bold-italics increase it by $20 \%$ and $13 \%$, respectively. Changing the font

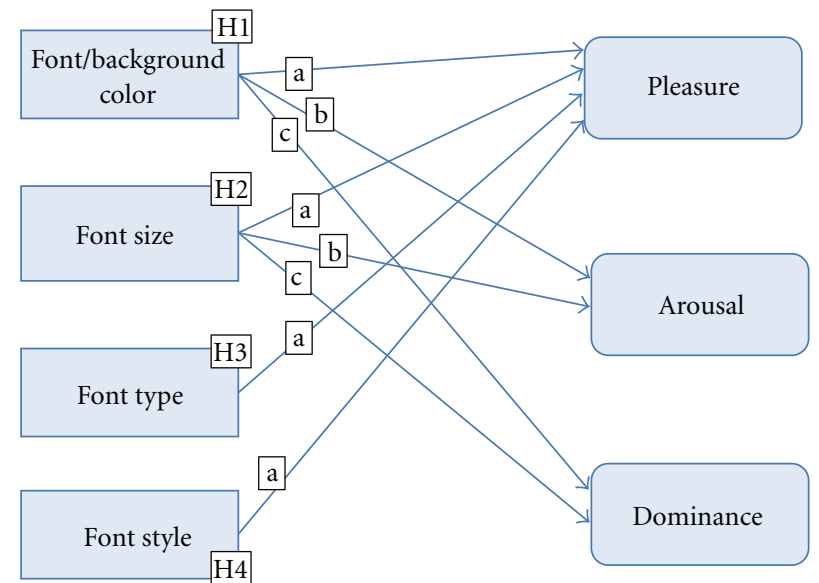

FIGURE 5: The proposed model for the relations between the independent variables on the three emotional dimensions "Pleasure", "Arousal", and "Dominance".

style elements increases the "Pleasure" dimension (except in the case of bold).

Times New Roman. in this case, all font style variations decrease "Pleasure": bold text by $31 \%$, italics by $19 \%$, and bold-italics by $21 \%$.

\section{Discussion and Modeling of the Results}

Following the ANOVA of the results described above, we can propose a general model (Figure 5) for the relations between the independent variables on the three emotional dimensions "Pleasure," "Arousal," and "Dominance."

Hypothesis 1. The scatter of "Pleasure," "Arousal," and "Dominance" on each color combination is presented in Figure 6, which facilitates the comparison of their mean values and the discussion of the current results.

The major findings are as follows.

(i) Not any emotional extremes were observed.

(ii) Black text on white background, white on blue, and green on yellow combinations were rated as the most pleasant combinations.

(iii) Black on white is considered as the least arousing combination. 


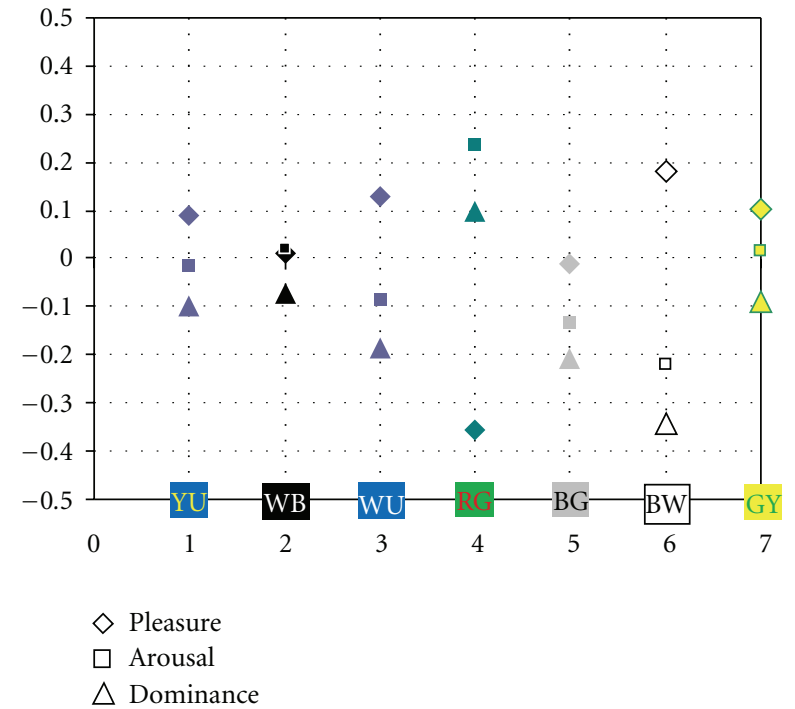

Figure 6: The mean values of each color combination for "Pleasure", "Arousal", and "Dominance". The code numbers in $X$ axis are 1 for Yellow on Blue, 2 for White on Black, 3 for White on Blue, 4 for Red on Green, 5 for Black on Gray, 6 for Black on White, and 7 for Green on Yellow.

(iv) Red text font color on green background is the least pleasant and most arousing color combination.

Moreover, Hall and Hanna [45] showed that color combinations with higher levels of contrast, including the color blue, receive higher ratings in readability and aesthetics. The high-contrast-color combinations (black on white, white on black, yellow on blue, and white on blue) lead to higher values on the "Pleasure" dimension (H1a) and lower values on the "Arousal" and "Dominance" (H1b and H1c), respectively. But there are some deviations from the expected values.

(i) White on black (high-contrast color combination) results in higher values on the "Arousal" dimension than the expected.

(ii) Black on gray (low contrast color combination) results in lower values on the "Arousal" and "Dominance" dimensions.

(iii) Green on yellow (low contrast color combination) results in higher values on the "Pleasure" dimension and lower values on the "Dominance". This effect is in agreement with similar findings by Laarni [56].

The current results are consistent with those presented in [56]: (a) the font/background color combinations affect "Pleasure", "Arousal," and "Dominance". (b) "Red on green" is considered as the most unpleasant combination and also elicited the highest arousal reactions. (c) "Black on white" has the lowest mean arousal level. (d) "White on blue" and "green on yellow" are the most pleasant combinations. Both the results of this work and those in [56] were derived with the SAM test, but based on participants with linguistic and cultural differences, indicating that the affect of text/background color combinations used in typography on PAD is possibly language and culture independent. This assumption has to be further examined (using the same or similar methodology) across other non-Western languages and cultures. There are indications for the consistency of the results in Arabic language [74, 76]. Also Cyr et al. [77] concluded that there are some similarities in the web site color scheme reactions across cultures (Canadian, German, and Japanese), but some differences have to be considered. For instance, the three cultures tended to dislike the yellow color scheme websites. Germans had the most pronounced preference for the blue color scheme, and Canadians appreciated the gray color scheme more than Germans and Japanese.

Hypothesis 2. Based on the results of the current work (Table 2), we can model the PAD relationship on text size using the following equation:

$$
f_{\{d\}}(s)=B_{3}^{\{d\}} \cdot s^{3}+B_{2}^{\{d\}} \cdot s^{2}+B_{1}^{\{d\}} \cdot s+\text { Intercept }^{\{d\}},
$$

where $d: P, A$, or $D ; P$ : Pleasure in $[-1,1]$; $A$ : Arousal in $[-1,1] ; D$ : Dominance in $[-1,1] ; B_{1}, B_{2}$, and $B_{3}$ are the polynomial coefficients; and $s$ : Font size in px.

Table 7 presents the constants of the polynomial fittings after applying the statistical package Origin 8.0 [78] on (2). The results indicate that "Pleasure" versus "Font Size" can be described using a second-degree equation, and "Arousal" and "Dominance" can be described using a thirddegree equation. The adjusted R-square is the measure of correctness of the fitting.

The application of the above model for "Pleasure", "Arousal", and "Dominance" versus "Font Size" is graphically displayed in Figures 7(a), 7(b), and 7(c), respectively.

The above results are consistent with those of Bernardini et al. [68], who noted that the preference emotional level decreases not only using smaller font size but also using larger font size.

Hypotheses 3 and 4. In our study we found that only the "Pleasure" dimension was affected by font style and font type. But we have to take into account the fact that the variations of the emotional dimensions were not extreme on the variations of font type and font style.

7.1. Modeling the Mapping of Typographic Elements into Emotional States. The results of this work indicate that the values of the typographic elements can have either discrete (e.g., font style) or continuous values (e.g., font size). If $P$ (Pleasure), $A$ (Arousal), and $D$ (Dominance) are used as the dependent variables, the mapping of the typographic elements into emotional states can be modeled using linear equations (e.g., $P=a_{1}^{P} \cdot x+b_{P}$ ) or single variable polynomial equations of the type

$$
\begin{aligned}
f_{\{d\}}(x)= & a_{n}^{\{d\}} \cdot x^{n}+\cdots+a_{3}^{\{d\}} \cdot x^{3}+a_{2}^{\{d\}} \cdot x^{2} \\
& +a_{1}^{\{d\}} \cdot x+b_{\{d\}}
\end{aligned}
$$




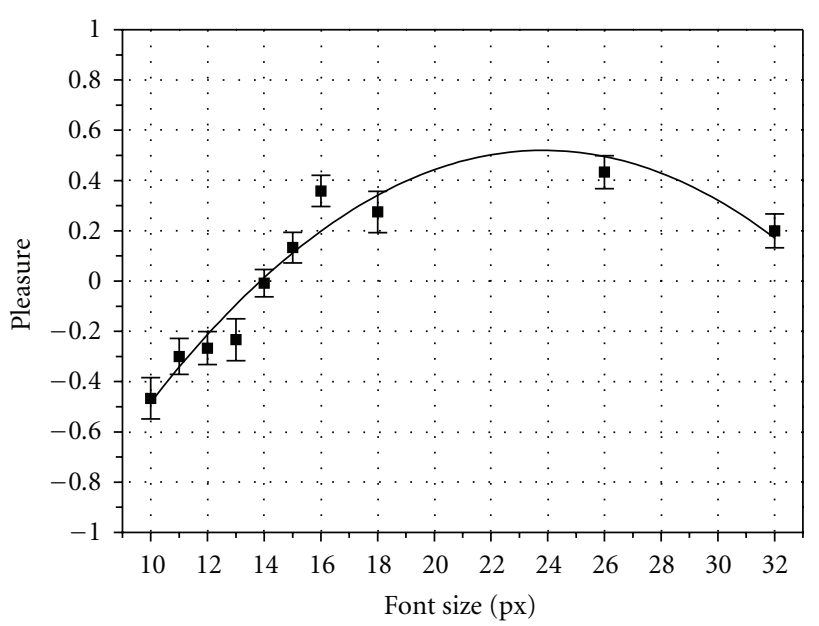

(a)

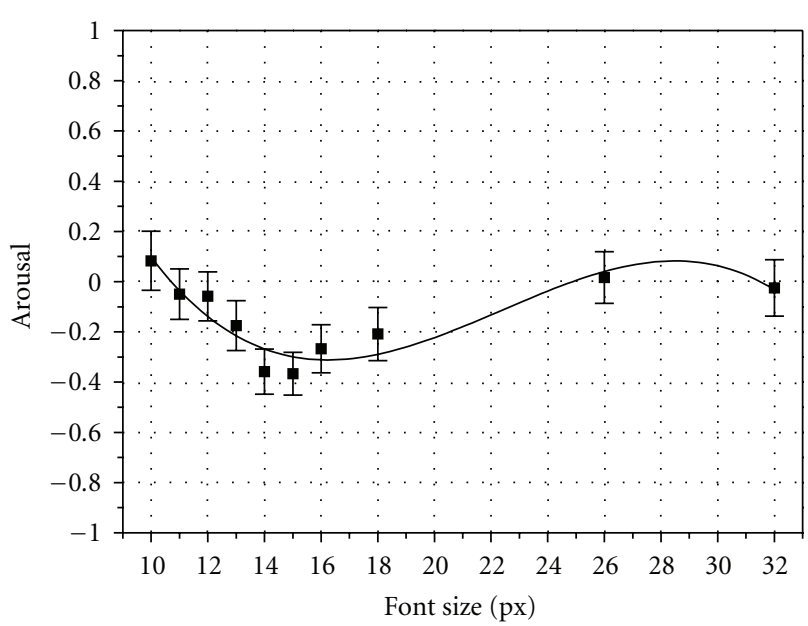

(b)

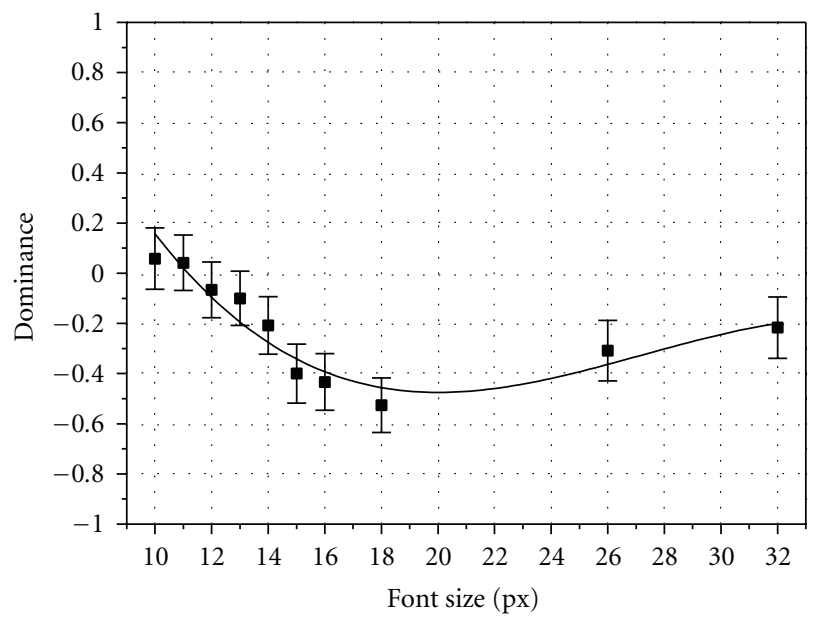

(c)

Figure 7: (a) Modeling "Pleasure" versus "Font Size" with (2). (b) Modeling "Arousal" versus "Font Size" with (2). (c) Modeling "Dominance" versus "Font Size" with (2). or multiple variable polynomial equations of the form

$$
\begin{aligned}
f_{\{d\}}(x)= & a_{n}^{\{d\}} \cdot x_{n}+\cdots+a_{3}^{\{d\}} \cdot x_{3}+a_{2}^{\{d\}} \cdot x_{2} \\
& +a_{1}^{\{d\}} \cdot x_{1}+b_{\{d\}} .
\end{aligned}
$$

Equations (3) and (4) can be described in the form of

$$
E=A \cdot X+B
$$

where

$$
\begin{array}{ll}
E=\left[\begin{array}{c}
P \\
A \\
D
\end{array}\right], & X=\left[\begin{array}{c}
x \\
x^{2} \\
x^{3} \\
\vdots \\
x^{n}
\end{array}\right] \quad \text { or } \quad X=\left[\begin{array}{c}
x_{1} \\
x_{2} \\
x_{3} \\
\vdots \\
x_{n}
\end{array}\right], \\
A & =\left[\begin{array}{ccccc}
a_{1}^{P} & a_{2}^{P} & a_{3}^{P} & \cdots & a_{n}^{P} \\
a_{1}^{A} & a_{2}^{A} & a_{3}^{A} & \cdots & a_{n}^{A} \\
a_{1}^{D} & a_{2}^{D} & a_{3}^{D} & \cdots & a_{n}^{D}
\end{array}\right],
\end{array}
$$

and $E(3 \times 1)$ describes the three dimensions of "Pleasure", "Arousal," and "Dominance". $X(n \times 1)$ describes the independent variable(s). $A(3 \times n)$ describes the factors of the independent variable $(s) . B(3 \times 1)$ is the offset.

The above equations are valid for continuous values of typographic elements. In the case where the values of the typographic elements are discrete, (5) is converted into

$$
E_{i}=B_{i}
$$

where

$$
E_{i}=\left[\begin{array}{c}
P_{i} \\
A_{i} \\
D_{i}
\end{array}\right], \quad B_{i}=\left[\begin{array}{c}
b_{i, P} \\
b_{i, A} \\
b_{i, D}
\end{array}\right]
$$

$i \in Q$ where $Q$ corresponds to the values of the typographic elements.

These two cases can be explained using the following examples.

Example 1. Suppose the model is described by (2)

$$
\begin{aligned}
P= & -0.00523 \cdot x^{2}+0.24942 \cdot x-2.45287 \\
A= & -0.00042711 \cdot x^{3}+0.02866 \cdot x^{2} \\
& -0.59264 \cdot x+3.58459 \\
D= & 0.000199863 \cdot x^{3}+0.01631 \cdot x^{2} \\
& -0.41265 \cdot x+2.85307
\end{aligned}
$$


This modeling can be represented using (5) by

$$
\begin{gathered}
E=\left[\begin{array}{l}
P \\
A \\
D
\end{array}\right], \quad X=\left[\begin{array}{l}
x \\
x^{2} \\
x^{3}
\end{array}\right], \\
A=\left[\begin{array}{ccc}
0.24942 & -0.00523 & 0 \\
-0.59264 & 0.02866 & -0.00042711 \\
-0.41265 & 0.01631 & 0.000199863
\end{array}\right], \\
B=\left[\begin{array}{c}
-2.45287 \\
3.58459 \\
2.85307
\end{array}\right] .
\end{gathered}
$$

Example 2. Suppose the model describing the reader's emotional state variations on font style elements on Arial is

(i) Pleasure-plain: 0, bold: -0.03 , italics: 0.2, bolditalics: 0.13;

(ii) Arousal-plain: 0, bold: 0, italics: 0, bold-italics: 0;

(iii) Dominance-plain: 0, bold: 0, italics: 0, bold-italics: 0 .

The modeling can be represented using (7) as:

$$
\begin{gathered}
{\left[\begin{array}{c}
E_{p} \\
E_{b} \\
E_{i} \\
E_{b i}
\end{array}\right]=\left[\begin{array}{c}
B_{p} \\
B_{b} \\
B_{i} \\
B_{b i}
\end{array}\right], \quad E_{p}=\left[\begin{array}{c}
P_{p} \\
A_{p} \\
D_{p}
\end{array}\right], \quad E_{b}=\left[\begin{array}{c}
P_{b} \\
A_{b} \\
D_{b}
\end{array}\right],} \\
E_{i}=\left[\begin{array}{c}
P_{i} \\
A_{i} \\
D_{i}
\end{array}\right], \quad E_{b i}=\left[\begin{array}{c}
P_{b i} \\
A_{b i} \\
D_{b i}
\end{array}\right], \quad B_{p}=\left[\begin{array}{c}
0 \\
0 \\
0
\end{array}\right], \\
B_{b}=\left[\begin{array}{c}
0.2 \\
-0.03 \\
0
\end{array}\right], \quad B_{i}=\left[\begin{array}{c}
0.13 \\
0
\end{array}\right] .
\end{gathered}
$$

\section{Conclusions}

In this work we have described the results of an experimental study towards modeling the reader's emotional state variations derived by the typographic elements in documents. Our main motivation was the effective acoustic rendition of the typographic elements using expressive speech synthesis. Based on the experimental results we propose a set of mapping rules on how typographic elements, such as font style (plain, italics, bold, and bold-italics), and font elements (font/background color combinations, font type, and font size) affect the reader's emotional state. Our study was based on the dimensional approach of the emotions (namely "Pleasure", "Arousal", and "Dominance") and the experimental procedure was designed and implemented according to IAPS experimental guidelines, using the Self-Assessment Manikin test [75]. There are indications that emotional states have similar affection and variations across language and culture. This assumption derives from the comparison of our experimental results with those from similar studies for other languages and cultures.

Some major findings of this work are as follows. As the font size increases up to $26 \mathrm{px}$ there is an increase on the "Pleasure" dimension. From this value and higher there is a decrease. In contradiction, "Arousal" and "Dominance" decrease up to $15 \mathrm{px}$ and $18 \mathrm{px}$, respectively. Beyond these values there is an increase in both dimensions. The results denoted that only "Pleasure" is affected by the font type. Times New Roman is considered more pleasant than Arial (23\% increase of "Pleasure" using plain text), and only "Pleasure" is affected by font style elements.

Moreover, we introduced a model for the quantitative representation of the results. We are using the polynomial regression for the case of font size variations (continuous values) and simple percentage variations for the case of font type and font style (discrete values). Based on this modeling and the need of implementing the rules for the automated reader's emotional state extraction [12], we propose a generic mathematical description on how the variations of the typographic elements can be mapped into emotional state values. Future work can investigate and model other combinations of typographic elements (e.g., study a greater number of color combinations and font type/font style) and even include the layout layer or/and the logical layer of text documents.

The model we have proposed is not scalable. Moreover, it matters whether the text is written on, for example, a paper box, a newspaper, or on a bill board, on how the typography is perceived. Thus, future work has to explore the context effects on how the typography of a text presented on various forms of a computer screen is perceived.

Kouroupetroglou et al. [10] proposed the use of the presented model (or models that derive by the proposed future work) for the automated reader's emotional state extraction and annotation of an Open Document Format (ODF) document. Thus, we are able to automatically add this information (metadata) to an .odt file. This information can be useful to, for example, a system that can automatically aesthetically emotionally rate/rank a specific block of text or even the entire document (obviously using the presented or even more complex rules) in order to propose (to the designer and/or developer) the improvement of the visual presentation of the document.

Another proposed application is that, by adding the metadata from the visual modality (reader's emotional state), we are able to convey this information into other modalities, acoustic and/or haptic. For example, combining this model (typography to emotional state) and the acoustic model introduced by Schröder [79] (emotional state to speech), we are able to convey the typographic information of a document to the acoustic modality using prosody manipulation of a Text-to-Speech system, as presented in the proposed architecture in $[9,80]$. 


\section{Acknowledgments}

The authors would like to thank Kalliopi Ikospentaki for her valuable contribution and help during the statistical analysis of the data. The work described in this paper has been funded by (a) the European Social Fund and Hellenic National resources under the HOMER project of the PENED Programme, Greek General Secretariat of Research and Technology and (b) the National and Kapodistrian University of Athens, Special Account for Research Grants.

\section{References}

[1] F. Fourli-Kartsouni, K. Slavakis, G. Kouroupetroglou, and S. Theodoridis, "A Bayesian network approach to semantic labelling of text formatting in XML corpora of documents," in Proceedings of the Human-Computer Interaction International (HCII '07), vol. 4556 of Lecture Notes in Computer Science (LNCS), pp. 299-308, 2007.

[2] G. Xydas and G. Kouroupetroglou, "Text-to-speech scripting interface for appropriate vocalisation of e-texts," in Proceedings of the European Conference on Speech Communication and Technology (EUROSPEECH '01), pp. 2247-2250, 2001.

[3] G. Xydas and G. Kouroupetroglou, "Augmented auditory representation of e-texts for text-to-speech systems," in Proceedings of the 4th International Conference on Text, Speech and Dialogue (TSD '01), vol. 2166 of Lecture Notes in Artificial Intelligence (LNAI), pp. 134-141, 2001.

[4] G. Xydas, D. Spiliotopoulos, and G. Kouroupetroglou, "Modelling emphatic events from non-speech aware documents in speech based user interfaces," in Proceedings of the 10th International Conference on Human-Computer Interaction (HCII '03), pp. 806-810, Lawrence Erlbaum Associates, Mahwah, NJ, USA, 2003.

[5] G. Xydas, V. Argyropoulos, T. Karakosta, and G. Kouroupetroglou, "An experimental approach in recognizing synthesized auditory components in a non-visual interaction with documents," in Proceedings of the 11th International Conference on Human-Computer Interaction (HCII '05), vol. 3, pp. 411-420, Las Vegas, Nev, USA, 2005.

[6] D. Spiliotopoulos, G. Xydas, G. Kouroupetroglou, V. Argyropoulos, and K. Ikospentaki, "Auditory universal accessibility of data tables using naturally derived prosody specification," Universal Access in the Information Society, vol. 9, no. 2, pp. 169-183, 2010.

[7] D. Spiliotopoulos, G. Xydas, G. Kouroupetroglou, and V. Argyropoulos, "Experimentation on spoken format of tables in auditory user interfaces," in Proceedings of the 11th International Conference on Human-Computer Interaction (HCII '05), pp. 361-370, Las Vegas, Nev, USA, 2005.

[8] D. Spiliotopoulos, G. Xydas, and G. Kouroupetroglou, "Diction based prosody modeling in table-to-speech synthesis," in Proceedings of the International Conference on Text, Speech and Dialogue (TSD '05), vol. 3658 of Lecture Notes in Computer Science (LNCS), pp. 294-301, 2005.

[9] G. Kouroupetroglou and D. Tsonos, "Multimodal accessibility of documents," in Advances in Human-Computer Interaction, pp. 451-470, I-Tech Education and Publishing, Vienna, Austria, 2008.

[10] G. Kouroupetroglou, D. Tsonos, and E. Vlahos, "DocEmoX: a system for the typography-derived emotional annotation of documents," in Proceedings of the International Conference on
Universal Access in Human-Computer Interaction, vol. 5616 of Lecture Notes in Computer Science (LNCS), pp. 550-558, 2009.

[11] D. Tsonos and G. Kouroupetroglou, "Accessibility of board and presentations in the classroom: a design-for-all approach," in Proceedings of the 4th IASTED International Conference on Telehealth and Assistive Technologies (TAT '08), pp. 13-18, ACTA Press, April 2008.

[12] D. Tsonos, K. Ikospentaki, and G. Kouroupetrolgou, “Towards modeling of readers' emotional state response for the automated annotation of documents," in Proceedings of the IEEE World Congress on Computational Intelligence (WCCI '08), pp. 3253-3260, Hong Kong, 2008.

[13] D. Freitas and G. Kouroupetroglou, "Speech technologies for blind and low vision persons," Technology and Disability, vol. 20, no. 2, pp. 135-156, 2008.

[14] K. Fellbaum and G. Koroupetroglou, "Principles of electronic speech processing with applications for people with disabilities," Technology and Disability, vol. 20, no. 2, pp. 55-85, 2008.

[15] M. McLuhan and Q. Fiore, The Medium is the Message, Gingko Press, Berkeley, Calif, USA, 2005.

[16] O. Briggs, S. Champeon, E. Costello, and M. Patterson, Cascading Style Sheets: Separating Content from Presentation, Friends of ED, 2nd edition, 2004.

[17] W. Tracy, Letters of Credit: A View of Type Design, Gordon Fraser, Jersey City, NJ, USA, 1986.

[18] P. Ekman, "An argument for basic emotions," Cognition and Emotion, vol. 6, no. 3, pp. 169-200, 1992.

[19] M. M. Bradley, "Measuring emotion: the self-assessment manikin and the semantic differential," Journal of Behavior Therapy and Experimental Psychiatry, vol. 25, no. 1, pp. 49-59, 1994.

[20] M. M. Bradley and P. J. Lang, "Affective reactions to acoustic stimuli," Psychophysiology, vol. 37, no. 2, pp. 204-215, 2000.

[21] C. Dormann, "Affective experiences in the home: measuring emotions," in Proceedings of the International Conference on Home Oriented Informatics and Telematics (HOIT '03), Calif, USA, 2003.

[22] T. Partala and V. Surakka, "The effects of affective interventions in human-computer interaction," Interacting with Computers, vol. 16, no. 2, pp. 295-309, 2004.

[23] K. R. Scherer, "What are emotions? and how can they be measured?" Social Science Information, vol. 44, no. 4, pp. 693727, 2005.

[24] R. W. Picard, "Affective computing," Tech. Rep. 321, M.I.T Media Laboratory Perceptual Computing Section, 1995.

[25] P. J. Lang, "The cognitive psychophysiology of emotion: fear and anxiety," in Anxiety and the Anxiety Disorder, A. Tuma and J. Maser, Eds., pp. 131-170, Lawrence Erlbaum, Mahwah, NJ, USA, 1985.

[26] J. D. Morris, "Observations SAM: the self-assessment manikin- An efficient cross-cultural measurement of emotional response," Journal of Advertising Research, pp. 63-68, 1995.

[27] R. P. Bagozzi, M. Gopinath, and P. U. Nyer, "The role of emotions in marketing," Journal of the Academy of Marketing Science, vol. 27, no. 2, pp. 184-206, 1999.

[28] J. Posner, J. A. Russell, and B. S. Peterson, "The circumplex model of affect: an integrative approach to affective neuroscience, cognitive development, and psychopathology," Development and Psychopathology, vol. 17, no. 3, pp. 715-734, 2005.

[29] T. Bänziger, V. Tran, and K. Scherer, "The Geneva Emotion Wheel: a tool for the verbal report of emotional reactions," 
in Proceedings of the Conference of the International Society for Research on Emotions (ISRE '05), Bari, Italy, 2005.

[30] A. C. Boucouvalas, "Real time text-to-emotion engine for expressive Internet communications," in Being There: Concepts, Effects and Measurement of User Presence in Synthetic Environments, G. Riva, F. Davide, and W. IJsselsteijn, Eds., vol. 5 of Emerging Communication: Studies on New Technologies and Practices in Communication, pp. 306-318, IOS Press, Amsterdam, The Netherlands, 2003.

[31] X. Zhe, D. John, and A. C. Boucouvalas, "Text-to-Emotion Engine: tests of user preferences," in Proceedings of the EEE International Symposium on Consumer, 2002.

[32] H. Liu, H. Lieberman, and T. Selker, "A model of textual affect sensing using real-world knowledge," in Proceedings of the International Conference on Intelligent User Interfaces, pp. 125-132, Miami, Fla, USA, January 2003.

[33] C.-H. Wu, Z.-J. Chuang, and Y.-C. Lin, "Emotion recognition from text using semantic labels and separable mixture models," ACM Transactions on Asian Language Information Processing, vol. 5, no. 2, pp. 165-182, 2006.

[34] H. J. Min and J. C. Park, "Representing emotions with linguistic acuity," in Proceedings of the Conference on Intelligent Text Processing and Computational Linguistics, vol. 4394 of Lecture Notes in Computer Science (LNCS), pp. 348-360, 2007.

[35] Z. Yang, L. Li, and D. Zhang, "Embodiment of text based on virtual robotic avatar," in Proceedings of the IEEE International Conference on Robotics and Biomimetics (ROBIO '07), pp. 1285-1289, December 2007.

[36] A. J. Gill, D. Gergle, R. M. French, and J. Oberlander, "Emotion rating from short blog texts," in Proceedings of the 26th Annual SIGCHI Conference on Human Factors in Computing Systems (CHI '08), pp. 1121-1124, ACM Press, New York, NY, USA, 2008.

[37] J. T. Hancock, C. Landrigan, and C. Silver, "Expressing emotion in text-based communication," in Proceedings of the 25th SIGCHI Conference on Human Factors in Computing Systems (CHI '07), pp. 929-932, ACM Press, New York, NY, USA, May 2007.

[38] C. O. Alm, Affect in text and speech, Ph.D. thesis, VDM Verlag Dr. Müller, Saarbrücken, Germany, 2009.

[39] J. Read, "Using emoticons to reduce dependency in machine learning techniques for sentiment classification," in Proceedings of the Association for Computational Linguistics Student Research Workshop (ACL '05), pp. 43-48, 2005.

[40] R. Cowie, E. Douglas-Cowie, B. Appolloni, J. Taylor, A. Romano, and W. Fellenz, "What a neural net needs to know about emotion words," in Computational Intelligence and Applications, N. Mastorakis, Ed., pp. 109-114, World Scientific \& Engineering Society Press, River Edge, NJ, USA, 1999.

[41] S. Owsley, S. Sood, and J. K. Hammond, "Domain specific affective classification of documents," in Proceedings of the AAAI of the Spring Symposia on Computational Approaches to Analyzing Weblogs (CAAW'06), 2006.

[42] K. H. Y. Lin, C. Yang, and H. H. Chen, "What emotions do news articles trigger in their readers?" in Proceedings of the 30th Annual International ACM SIGIR Conference on Research and Development in Information Retrieval (SIGIR '07), pp. 733734, ACM Press, New York, NY, USA, July 2007.

[43] C. B. Mills and L. J. Weldon, "Reading Text from computer screens," Computing Surveys, vol. 19, no. 4, pp. 329-357, 1987.

[44] V. Eglin and S. Bres, "Document page similarity based on layout visual saliency: application to query by example and document classification," in Proceedings of the 7th International
Conference on Document Analysis and Recognition (ICDAR '03), vol. 2, p. 1208, IEEE Computer Society, 2003.

[45] R. H. Hall and P. Hanna, "The impact of web page textbackground colour combinations on readability, retention, aesthetics and behavioural intention," Behaviour and Information Technology, vol. 23, no. 3, pp. 183-195, 2004.

[46] M. C. Dyson and G. J. Kipping, "The legibility of screen formats: are three columns better than one?" Computers and Graphics, vol. 21, no. 6, pp. 703-712, 1997.

[47] N. Holmberg, Eye movement patterns and newspaper design factors. An experimental approach, M.S. thesis, Lund University Cognitive Science, Lund, Sweden, 2004.

[48] K. Holmqvist and C. Wartenberg, "The role of local design factors for newspaper reading behaviour-an eye-tracking respective," Lund University Cognitive Studies, vol. 127, pp. 121, 2005.

[49] C. Wartenberg and K. Holmqvist, Daily Newspaper LayoutDesigners' Predictions of Readers' Visual Behaviour-A Case Study, Lund University Cognitive Studies, Lund, Sweden, 2005.

[50] A. Hill and L. V. Scharff, "Readability of screen displays with various foreground/background color combinations, font styles, and font types," in Proceedings of the 11th National Conference on Undergraduate Research (NCUR '97), vol. 2, pp. 742-746, Austin, Tex, USA, 1997.

[51] S. J. Harrington, P. Roetling, J. F. Naveda, R. P. Jones, and N. Thakkar, "Aesthetic measures for automated document layout," in Proceedings of the ACM Symposium on Document Engineering (DocEng '04), pp. 109-111, ACM Press, New York, NY, USA, October 2004.

[52] T. Porat, R. Liss, and N. Tractinsky, "E-stores design: the influence of E-store design and product type on consumers' emotions and attitudes," in Proceedings of the HumanComputer Interaction (HCII '07), vol. 4553 of Lecture Notes in Computer Science (LNCS), pp. 712-721, 2007.

[53] F. Calisir, M. Eryazici, and M. R. Lehto, "The effects of text structure and prior knowledge of the learner on computerbased learning," Computers in Human Behavior, vol. 24, no. 2, pp. 439-450, 2008.

[54] J. Borchers, O. Deussen, A. Klingert, and C. Knörzer, "Layout rules for graphical Web documents," Computers and Graphics, vol. 20, no. 3, pp. 415-426, 1996.

[55] J. Éthier, P. Hadaya, J. Talbot, and J. Cadieux, "Interface design and emotions experienced on B2C Web sites: empirical testing of a research model," Computers in Human Behavior, vol. 24, no. 6, pp. 2771-2791, 2008.

[56] J. Laarni, "Effects of color, font type and font style on user preferences," in Proceedings of the Human-Computer Interaction International (HCII '03), C. Stephanidis, Ed., pp. 31-32, Heraklion, Greece, 2003.

[57] F. Birren, Color \& Human Response: Aspects of Light and Color, Bearing on the Reactions of Living Things and the Welfare of Human Beings, John Wiley \& Sons, New York, NY, USA, 1984.

[58] J. A. Sánchez, I. Kirschning, J. C. Palacio, and Y. Ostróvskaya, "Towards mood-oriented interfaces for synchronous interaction," in Proceedings of the Latin American Conference on Human-Computer interaction (CLIHC '05), vol. 124, pp. 1-7, ACM Press, New York, NY, USA, 2005.

[59] J. A. Sánchez, N. P. Hernández, J. C. Penagos, and Y. Ostróvskaya, "Conveying mood and emotion in instant messaging by using a two-dimensional model for affective states," in Proceedings of the 7th Symposium on Human Factors in Computing Systems (IHC '06), pp. 66-72, ACM Press, New York, NY, USA, 2006. 
[60] T. Saari, M. Turpeinen, J. Laarni, N. Ravaja, and K. Kallinen, "Emotionally loaded mobile multimedia messaging," in Proceedings of the 3rd International Conference on Entertainment Computing (ICEC '04), vol. 3166 of Lecture Notes in Computer Science (LNCS), pp. 476-486, Eindhove, The Netherlands, 2004.

[61] K. Larson, “The technology of text," IEEE Spectrum, vol. 44, no. 5, pp. 26-31, 2007.

[62] A. Kalra and K. Karahalios, "TextTone: expressing emotion through text," in Proceedings of the International Conference on Human-Computer Interaction (INTERACT '05), vol. 3585 of Lecture Notes in Computer Science (LNCS), pp. 966-969, Rome, Italy, 2005.

[63] J. Ohene-Djan and R. Shipsey, "E- subtitles: emotional subtitles as a technology to assist the deaf and hearing-impaired when learning from television and film," in Proceedings of the 6th International Conference on Advanced Learning Technologies (ICALT '06), pp. 464-466, July 2006.

[64] J. Ohene-Djan, J. Wright, and K. Combie-Smith, "Emotional subtitles: a system and potential applications for deaf and hearing impaired people," in Proceedings of the Conference and Workshop on Assistive Technologies for People with Vision and Hearing Impairments: Assistive Technology for All Ages (CVHI '07), p. 415, Granada, Spain, 2007.

[65] A. Yannicopoulou, "Visual aspects of written texts: preschoolers view comics," L1-Educational Studies in Language and Literature, vol. 4, no. 2-3, pp. 169-181, 2004.

[66] T. Rosenberger, Prosodic font: between the spoken and the written, Master of Media Arts and Sciences Thesis, Massachusetts Institute of Technology, 1988.

[67] T. Rosenberger and R. MacNeil, "Prosodic font: translating speech into graphics," in Proceedings of the ACM Conference on Computer-Human Interaction, pp. 252-253, Pittsburgh, Pa, USA, 1999.

[68] C. Bernardini, V. Ambrogi, G. Fardella, L. Perioli, and G. Grandolini, "How to improve the readability of the patient package leaflet: a survey on the use of colour, print size and layout," Pharmacological Research, vol. 43, no. 5, pp. 437-443, 2001.

[69] M. L. Bernard, B. S. Chaparro, M. M. Mills, and C. G. Halcomb, "Comparing the effects of text size and format on the readibility of computer-displayed Times New Roman and arial text," International Journal of Human Computer Studies, vol. 59, no. 6, pp. 823-835, 2003.

[70] J. Ling and P. Van Schaik, "The influence of font type and line length on visual search and information retrieval in web pages," International Journal of Human Computer Studies, vol. 64, no. 5, pp. 395-404, 2006.

[71] W3C, "Techniques for Accessibility Evaluation and Repair Tools," 2000, http://www.w3.org/TR/AERT\#color-contrast.

[72] W3C, Cascading Style Sheets Level 2 Revision 1 (CSS 2.1) Specification, W3C Candidate Recommendation, September 2009, http://www.w3.org/TR/CSS2/syndata.html\#values.

[73] K. E. Schmidt, M. Bauerly, Y. Liu, and S. Sridharan, "Web page aesthetics and performance: a survey and an experimental study," in Proceedings of the 8th Annual International Conference on Industrial Engineering-Theory, Applications and Practice, 2003.

[74] A. Alsumait, A. Al-Osaimi, and H. Alfedaghi, "Arab children's reading preference for different online fonts," in Proceedings of the International Conference on Human-Computer Interaction (HCII '09), vol. 5613 of Lecture Notes in Computer Science (LNCS), pp. 3-11, 2009.
[75] P. J. Lang, M. Bradley, and B. Culthbert, "International affective picture system (IAPS): instruction manual and affective ratings," Tech. Rep. A-6, The Center for Research in Psychophysiology, University of Florida, Gainsville, Fla, USA, 2005.

[76] A. Al-Badi and P. Mayhew, "Cultural perception of user interface of a website: comparative study between liberal and prescriptive cultures (Arab vs. British)," in Proceedings of the IADIS International Conference E-Society, Avila, Spain, 2004.

[77] D. Cyr, M. Head, and H. Larios, "Colour appeal in website design within and across cultures: a multi-method evaluation," International Journal of Human Computer Studies, vol. 68, no. 1-2, pp. 1-21, 2010.

[78] “Origin 8.0," 2008, http://www.originlab.com/index.aspx?s=8 $\& \operatorname{lm}=10$.

[79] M. Schröder, "Expressing degree of activation in synthetic speech," IEEE Transactions on Audio, Speech and Language Processing, vol. 14, no. 4, pp. 1128-1136, 2006.

[80] D. Tsonos and G. Kouroupetroglou, "A methodology for the extraction of reader' $s$ emotional state triggered from text typography," in Tools in Artificial Intelligence, pp. 439-455, ITech Education and Publishing, Vienna, Austria, 2008. 

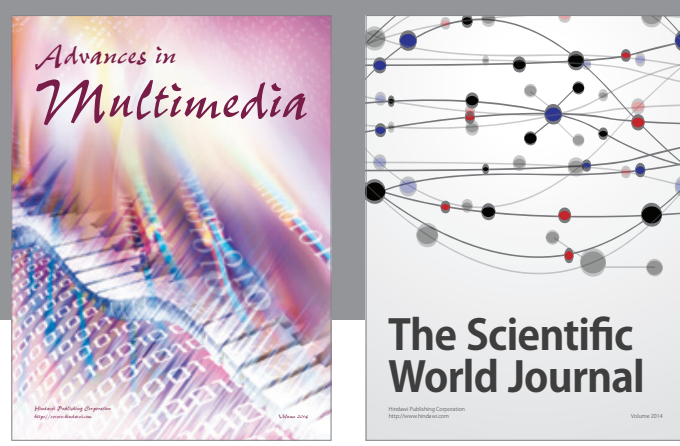

The Scientific World Journal
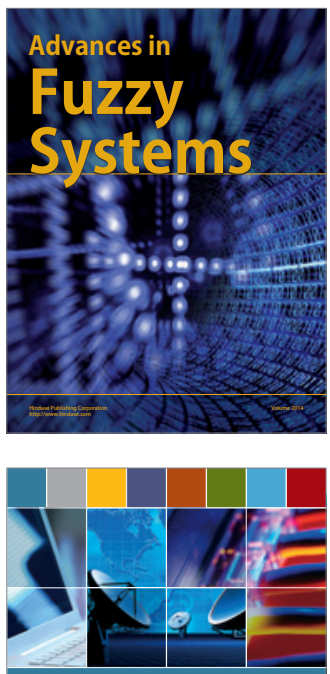

Computer Networks and Communications
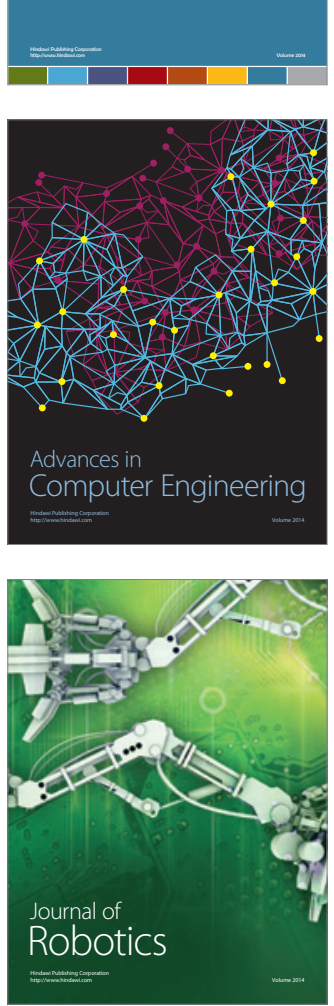
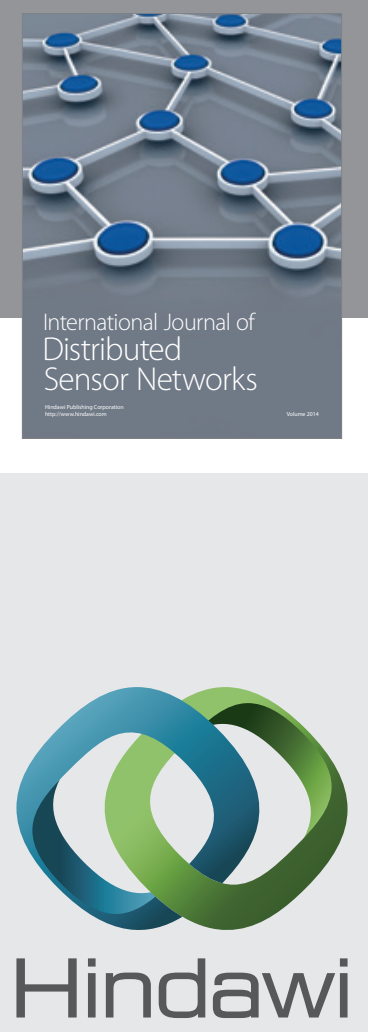

Submit your manuscripts at

http://www.hindawi.com
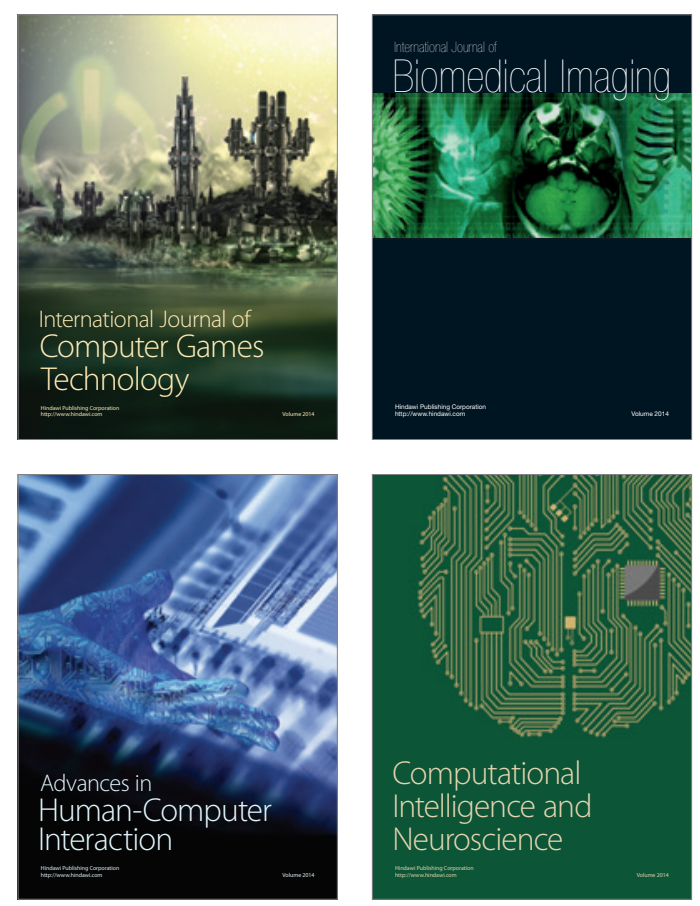
